\title{
Procesado de hojarasca en arroyos del Parque Nacional de la Sierra de Guadarrama (Madrid)
}

\author{
Carmen Casado ${ }^{1, *}$, Salvador Mollá ${ }^{1}$, José Manuel González², Neftalí Roblas ${ }^{3}$ y Enrique \\ Descals $^{4}$
}

${ }^{1}$ Dpto. Ecología, F. Ciencias, Universidad Autónoma de Madrid, C/ Darwin 2, Cantoblanco 28049 Madrid.

2 Dpto. Ciencias, Universidad Rey Juan Carlos, C/ Tulipán s/n, 28933 Móstoles Madrid.

${ }^{3}$ Centro de Investigaciones Ambientales de la Comunidad de Madrid. Ctra. M-607 km 20. 28760 Tres Cantos. Madrid.

${ }^{4}$ Instituto Mediterráneo de Estudios Avanzados, IMEDEA (CSIC), Miquel Marquès 21, 07190 Esporles, Mallorca.

* Autor responsable de la correspondencia: c.casado@uam.es

Recibido: 27/06/2014

Aceptado: 10/12/2014

\begin{abstract}
Leaf litter breakdown in streams of Sierra de Guadarrama National Park (Madrid)

The main goal of this study was to identify the main drivers of natural variability of leaf litter processing in six well preserved Mediterranean mountain streams in the Sierra de Guadarrama, through the analysis of the breakdown rates of alder litter, content and dynamics of leaf nutrients, and associated fungal and invertebrates communities. In general, breakdown rates of alder leaves, both exponential $\left(k=0.013 \pm 0.005 \mathrm{dd}^{-1}, k^{\prime}=0.041 \pm 0.011 \mathrm{~d}^{-1}\right)$ and lineal $(b=0.470 \pm 0.121 \%$ PSLC $\mathrm{dd}^{-1}, b^{\prime}=1.522 \pm 0.153 \%$ PSLC d $\left.{ }^{-1}\right)$ were very high. Variability in breakdown rates among streams was not correlated to environmental characteristics (discharge, slope or water temperature) neither to stream water characteristics (nutrient concentration, $\mathrm{pH}$, or conductivity). This may be due to the low environmental heterogeneity observed among these streams and also because the physical factors that could regulate this process were outweighed by biological processes that mask them. The highest breakdown rates were recorded in the coldest waters where the activity of decomposers aquatic fungi was very low (measured by sporulation rates, $98.36 \pm 148.84$ conidia $\mathrm{mg}^{-1} \mathrm{PSLC} \mathrm{d}^{-1}$ ), suggesting that consumer invertebrates played a key role in the faster leaf litter processing rates in these sites. Family richness $\left(13.67 \pm 3.44 \mathrm{fam}\right.$. $\left.\mathrm{bag}^{-1}\right)$ and macroinvertebrate density $\left(25.33 \pm 10.42\right.$ ind bag $\left.^{-1}\right)$, as well as shredder density $\left(16.17 \pm 7.44 \mathrm{ind} \mathrm{bag}^{-1}\right)$ did not show any relationship with the breakdown rates $(p>0.100$, neither linear nor exponential rates). A positive relationship was only found between percentage of leaf litter lost and abundance of Limnephilidae $(p<0.010)$, that pointed out a higher relevance of shredder traits than their total abundance. Limnephilidae are caddisfly shredders adapted to low thermal regimes, they have large body and are able to feed on unconditioned litter debris and poor colonized by aquatic hyphomycetes, which seem to exert a decisive control over leaf litter processing rate in this type of aquatic ecosystems. Therefore, we can suppose that in colder headwater mountain streams consumer macroinvertebrates (shredders) might have a major relevance in the processing of litter debris whereas decomposers (aquatic hyphomycetes) would play an increasing role in warmer streams.
\end{abstract}

Key words: Breakdown rate, litter bags, siliceous headwater mountain streams, macroinvertebrates, aquatic hyphomycetes.

\section{RESUMEN}

\section{Procesado de hojarasca en arroyos del Parque Nacional de la Sierra de Guadarrama (Madrid)}

El objetivo principal de este estudio fue evaluar y comparar la variabilidad natural del procesado de la hojarasca en seis arroyos y ríos mediterráneos de montaña de la Sierra de Guadarrama en buen estado de conservación, a través del análisis de las tasas de descomposición de hojarasca de aliso, su contenido y dinámica de nutrientes, y de las comunidades de hongos y macroinvertebrados asociadas. Las tasas de descomposición de la hojarasca de aliso, tanto exponenciales $\left(\mathrm{k}=0.013 \pm 0.005 d d^{-1}, \mathrm{k}^{\prime}=0.041 \pm 0.011 d^{-1}\right)$ como lineales $\left(b=0.470 \pm 0.121 \%\right.$ PSLC $d d^{-1}, \mathrm{~b}^{\prime}=1.522 \pm 0.153 \%$ $\left.P S L C d^{-1}\right)$ fueron en general muy altas. La variación en las tasas de descomposición entre los ríos no se correlacionó con 
las características ambientales de los arroyos (caudal, pendiente o temperatura del agua) ni con la composición del agua (nutrientes, $\mathrm{pH}$ o conductividad eléctrica). Esto pudo ser debido a la escasa heterogeneidad ambiental observada entre estos arroyos, y también a que los factores físicos que podrían regular este proceso se vean superados por procesos biológicos que los enmascaran. Las mayores tasas se registraron en las aguas más frías, donde la actividad descomponedora de los hongos

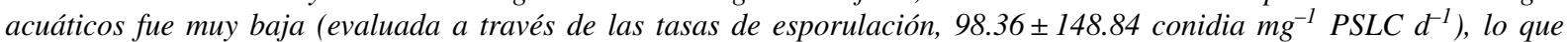
apuntaría a un papel más relevante de los consumidores del macrobentos en la velocidad de procesado de la hojarasca. La riqueza de familias de macroinvertebrados $\left(13.67 \pm 3.44\right.$ fam. bolsa $\left.^{-1}\right)$ y su densidad $\left(25.33 \pm 10.42\right.$ indiv. bolsa $\left.^{-1}\right)$, así como la densidad de desmenuzadores (16.17 \pm 7.44 indiv. bolsa $\left.{ }^{-1}\right)$, no mostraron relación con las tasas de descomposición lineales o exponenciales $(\mathrm{p}>0.100)$. Sólo se encontró una relación positiva entre el porcentaje de hojarasca perdida y la abundancia de limnefílidos ( $\mathrm{p}<0.010)$, lo que apunta a una mayor relevancia de las características de los desmenuzadores que de su abundancia. Se trata de tricópteros desmenuzadores adaptados a regímenes térmicos fríos, de gran tamaño y capaces de alimentarse de restos vegetales mal acondicionados y poco colonizados por hifomicetos acuáticos, que parecen ejercer un control decisivo en la velocidad del proceso de descomposición en este tipo de ecosistemas acuáticos. De este modo podemos suponer que en los ríos de montaña con aguas más frías los macroinvertebrados consumidores (desmenuzadores) tendrían una mayor relevancia en el procesado de los restos vegetales, mientras que en los ríos más cálidos sería mayor la importancia de los descomponedores (hifomicetos acuáticos).

Palabras clave: Tasas de descomposición, bolsas de hojarasca, arroyos de montaña silícea, macroinvertebrados, hifomicetos acuáticos.

\section{INTRODUCCIÓN}

Los ecosistemas en general, y especialmente los limnosistemas, están siendo degradados y la biodiversidad se está perdiendo a un ritmo no visto en la historia de la humanidad (Sandin \& Solomini, 2009). Esta fuerte presión ha sido reconocida por políticos y gestores y ha dado lugar a políticas como la Directiva Marco del Agua (DMA) (2000/60/CE), que tiene como objetivo proteger los recursos de agua superficial y mejorar el uso ecológicamente sostenible del agua. Esta Directiva establece que la calidad ecológica de las aguas superficiales debería ser cuantificada como "una expresión de la calidad de la estructura y el funcionamiento de los ecosistemas acuáticos asociados a las aguas superficiales", donde la estructura se refiere a los entornos físicos y químicos junto con la estructura biológica de las comunidades acuáticas de estos ecosistemas, principalmente los macroinvertebrados. Asume que su medida (a través de distintos indicadores estructurales) se relaciona directamente con el funcionamiento, es decir, que existe una correlación positiva entre la integridad estructural y funcional. Las medidas de la estructura se utilizan como sustitutos de propiedades funcionales a pesar de que la estructura y la función podrían en realidad no estar vinculadas (Sandin \& Solomini, 2009).
Aunque se han utilizado índices estructurales del estado ecológico e integridad de los ecosistemas fluviales durante más de 100 años, sólo recientemente se han desarrollado indicadores funcionales que completan esta visión y permiten la evaluación directa de la integridad funcional fluvial (Gessner \& Chauvet, 2002; Lecerf et al., 2006; Feio et al., 2010).

En los arroyos de cabecera que drenan cuencas forestales o que están bordeados por bosques de galería, la hojarasca de los ecosistemas terrestres circundantes es la mayor fuente de energía que mantiene a la red trófica fluvial. Por esta razón la descomposición de hojarasca es un proceso clave en estos ecosistemas (Wallace et al., 1997; Webster et al., 1999; Hall et al., 2000). La liberación del carbono y los nutrientes de las hojas está gobernada por una combinación compleja de mecanismos que implican factores abióticos, microorganismos y macroinvertebrados. Los descomponedores microbianos, principalmente los hongos hifomicetos acuáticos, degradan enzimáticamente las hojas y mejoran su contenido en nutrientes (Grattan \& Suberkropp, 2001) y los invertebrados desmenuzadores consumen, trituran, asimilan una parte, respiran y crecen en la hojarasca, contribuyendo así a su descomposición (Webster \& Benfield, 1986; Graça, 2001), y además, median en la transfe- 
rencia de materia y energía desde las hojas a otros niveles tróficos (Wallace et al., 1997).

Durante la última década, se ha propuesto y testado el proceso de descomposición de hojarasca como un método de evaluación directo de la integridad funcional de los ríos (Young et al., 2008; Riipinen et al., 2009; Feio et al., 2010) debido tanto a su naturaleza integradora como a su respuesta demostrada a diversas perturbaciones de origen humano (Danger \& Robson, 2004; Carlisle \& Clement, 2005; Elosegui et al., 2006), principalmente la eutrofización (Pozo et al., 2011) y la modificación del caudal (Niu \& Dudgeon, 2011). Estimar la integridad funcional de los ecosistemas fluviales a partir de las tasas de descomposición de hojarasca requiere conocer la variabilidad natural de la velocidad del proceso y sus valores de referencia, siempre dentro de regiones climáticas y geológicamente homogéneas (Casas et al., 2006; Casas et al., 2011; Menéndez et al., 2011), para cada tipo de río y en condiciones casi naturales o de referencia (por ejemplo, Reece \& Richardson, 1999). De encontrarse una variabilidad natural importante se reduciría la fiabilidad del método para detectar impactos antropogénicos (Pérez et al., 2011).

La descomposición de hojarasca se ha estudiado ampliamente en arroyos de bajo orden en los bosques templados mésicos de América del Norte y de Europa (Young et al., 2008; Tiegs et al., 2009; Riipinen et al., 2010). Aunque en los últimos 15 años se ha avanzado mucho en el conocimiento del proceso de descomposición en pequeños ríos de la Península Ibérica (Casas \& Gessner, 1999; Graça \& Canhoto, 2006; Menéndez et al., 2001; Sampaio et al., 2008; Martínez et al., 2013; entre otros), la información al respecto es escasa para grandes grupos de ríos ibéricos, como los existentes en zonas áridas y semiáridas (Casas et al., 2011) y en montañas mediterráneas (Pozo et al., 2011; Casas et al., 2013), así como los arroyos mediterráneos de montaña del Sistema Central peninsular, cuya evaluación del estado ecológico se ha basado exclusivamente en índices estructurales.

Ante esta situación, el objetivo principal de este estudio fue evaluar y comparar el proceso de descomposición de la hojarasca en varios arroyos de cabecera en estaciones no alteradas de un área geográfica y climática homogénea de la Península Ibérica, la vertiente meridional de la Sierra de Guadarrama de la Comunidad de Madrid. La variabilidad natural del proceso se examinó a través del análisis de las tasas de descomposición de hojarasca de Alnus glutinosa (L.) Gaertner, su contenido y dinámica de nutrientes, y de las co-

Tabla 1. Localización y características de las estaciones de muestreo. Location and characteristics of the studied sites.

\begin{tabular}{|c|c|c|c|c|c|c|}
\hline & $\begin{array}{c}\text { Río } \\
\text { Guadarrama }\end{array}$ & $\begin{array}{c}\text { Río } \\
\text { Navacerrada }\end{array}$ & $\begin{array}{c}\text { Río } \\
\text { Manzanares }\end{array}$ & $\begin{array}{l}\text { Arroyo } \\
\text { Mediano }\end{array}$ & $\begin{array}{c}\text { Arroyo Hoyo } \\
\text { Cerrado }\end{array}$ & $\begin{array}{c}\text { Arroyo Hoyo } \\
\text { Claveles }\end{array}$ \\
\hline Código de la estación & Gua & $\mathrm{Nav}$ & Man & Med & $\mathrm{HCe}$ & $\mathrm{HCl}$ \\
\hline Latitud & $40^{\circ} 46^{\prime} 28.08^{\prime \prime} \mathrm{N}$ & $40^{\circ} 45^{\prime} 25.60^{\prime \prime} \mathrm{N}$ & $40^{\circ} 46^{\prime} 16.45^{\prime \prime} \mathrm{N}$ & $40^{\circ} 47^{\prime} 4.36^{\prime \prime} \mathrm{N}$ & $40^{\circ} 52^{\prime} 32.00^{\prime \prime} \mathrm{N}$ & $40^{\circ} 52^{\prime} 2.00^{\prime \prime} \mathrm{N}$ \\
\hline Longitud & $4^{\circ} 3^{\prime} 57.11^{\prime \prime} \mathrm{O}$ & $3^{\circ} 59^{\prime} 30.44^{\prime \prime} \mathrm{O}$ & $3^{\circ} 54^{\prime} 38.31^{\prime \prime} \mathrm{O}$ & $3^{\circ} 49^{\prime} 54.43^{\prime \prime} \mathrm{O}$ & $3^{\circ} 55^{\prime} 2.00^{\prime \prime} \mathrm{O}$ & $3^{\circ} 54^{\prime} 37.00^{\prime \prime} \mathrm{O}$ \\
\hline Área de la Cuenca (Ha) & 175 & 532 & 1666 & 1129 & 178 & 173 \\
\hline Altitud (m) & 1390 & 1400 & 1220 & 1190 & 1380 & 1300 \\
\hline Pendiente del tramo (\%) & 29.2 & 21.9 & 13.6 & 20.0 & 27.5 & 24.7 \\
\hline Orden fluvial & 2 & 2 & 3 & 2 & 1 & 1 \\
\hline Anchura media $(\mathrm{m}) \pm \mathrm{SE}$ & $1.0 \pm 0.2$ & $3.3 \pm 0.6$ & $9.6 \pm 1.6$ & $5.5 \pm 1.0$ & $2.5 \pm 0.3$ & $1.1 \pm 0.2$ \\
\hline Profundidad media $(\mathrm{cm})( \pm \mathrm{SE})$ & $14.6 \pm 0.8$ & $12.1 \pm 1.3$ & $20.1 \pm 2.8$ & $19.8 \pm 0.6$ & $8.6 \pm 1.0$ & $9.4 \pm 1.1$ \\
\hline Cobertura riparia ( $\%$ ) & 66.6 & 86.0 & 64.1 & 83.4 & 81.1 & 82.0 \\
\hline Caudal $\left(1 \mathrm{~s}^{-1}\right) \pm \mathrm{SE}$ & $18.2 \pm 2.2$ & $60.5 \pm 13.8$ & $224.1 \pm 56.7$ & $83.4 \pm 31.3$ & $36.8 \pm 12.5$ & $11.5 \pm 2.7$ \\
\hline $\mathrm{IHF}$ & 61 & 71 & 85 & 73 & 59 & 56 \\
\hline QBR & 60 & 50 & 95 & 65 & 75 & 65 \\
\hline
\end{tabular}


munidades de hongos e invertebrados asociadas. Además se analizo la relación entre los diversos factores, tanto parámetros físico-químicos como actores biológicos, que pudieran estar influyendo en la variabilidad del proceso en estos arroyos.

\section{ÁREA DE ESTUDIO}

El estudio se llevó a cabo en la cabecera de seis arroyos de la ladera sur de la Sierra de Guadarrama en la Comunidad de Madrid: el río Guadarrama, río Navacerrada, río Manzanares y arroyo Mediano (pertenecientes a la cuenca del río Manzanares) y los arroyos Hoyo Cerrado y Hoyo Claveles (que pertenecen a la cuenca del río Lozoya). Su localización y características generales se recogen en la Figura 1 y en la Tabla 1. Los ríos de la Sierra de Guadarrama drenan rocas silíceas (principalmente granitos y granodioritas), están some- tidos a un clima mediterráneo templado-húmedo marcadamente continental y tienen un régimen nivopluvial muy influenciado por el deshielo pero con una fuerte sequía estival, ya que por la naturaleza poco permeable de sus cuencas presentan una rápida respuesta a episodios tormentosos, con rápidas crecidas y descensos de caudal $(\mathrm{Ca}-$ sado et al., 2011). Durante el ciclo hidrológico de estudio (octubre 2007-septiembre 2008) la temperatura media fue de $9.0^{\circ} \mathrm{C}$ y la precipitación total de $722 \mathrm{~mm}$. Todos los arroyos seleccionados pueden ser considerados estaciones de referencia de la categoría ríos de montaña silícea (tipo 11 en la tipología de ríos españoles según BOE 229/2008). Los ríos montañosos silíceos del Sistema Central son pequeños arroyos de montaña situados a elevadas altitudes, normalmente muy encajados, estrechos, poco profundos, con grandes afloramientos de roca madre y predominio de los hábitats de rápidos, poco desarrollo del medio

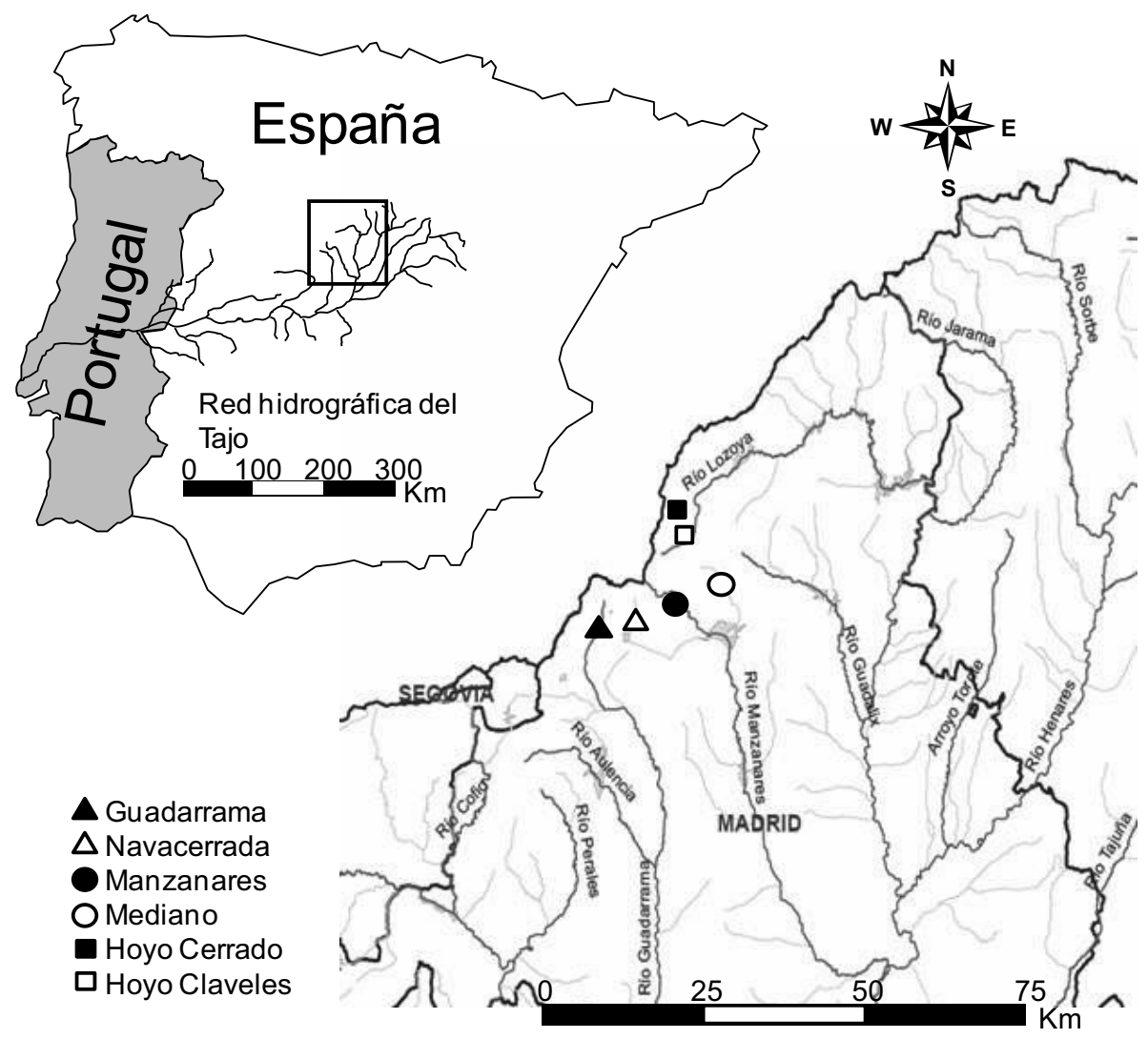

Figura 1. Localización del área de estudio y estaciones de muestreo. Location of the study area and sampling sites. 
hiporreico, escaso caudal y fuerte torrencialidad (Bordallo \& Casado, 2012). Debido a su elevada altitud (1190-1400 m.s.n.m.) las riberas de 4 de los 6 ríos estudiados están cubiertas por Pinus sylvestris L. y Quercus pyrenaica Willd. Los ríos Manzanares y Mediano presentan bosques de ribera dominados por sauces (principalmente Salix salvifolia, S. atrocinerea y $S$. purpurea) con algún pie aislado de fresno (Fraxinus angustifolia) o chopo (Populus nigra).

\section{METODOLOGÍA}

\section{Variables ambientales e hidroquímicas}

Los experimentos de descomposición se llevaron a cabo durante los meses de diciembre de 2007 y enero de 2008, coincidiendo con el pico estacional de la caída de las hojas y con la presencia de flujo continuo de agua. En cada una de las estaciones de los seis ríos estudiados se registró la temperatura del agua durante todo el periodo, a intervalos de una hora, mediante un registrador HOBO Pendant (Onset Computer Corporación, Bourne, Massachusetts). Además, en cada fecha de muestreo $(n=6)$ se midió in situ la conductividad, $\mathrm{pH}$ y oxígeno disuelto (sensor multiparamétrico WTW), y se tomaron muestras de agua para análisis de nutrientes. Estos análisis se realizaron con agua filtrada a través de filtros de fibra de vidrio (Whatman GF/F). La concentración de nitrato se determinó por el método del salicilato de sodio (Monteiro et al., 2003), la concentración de amonio por el del salicilato (Krom, 1980), el nitrito por el de la sulfanilamida, el fósforo reactivo soluble (PRS) por el del molibdato, y la alcalinidad por titulación hasta un $\mathrm{pH}$ final de 4.5 (APHA, 2005). También se estimó la anchura media del cauce inundado y su profundidad media a partir de seis mediciones realizadas en cada estación experimental. El caudal circulante se estimó por el método de los transectos, a partir de la medida de la velocidad de la corriente con un correntímetro (Global Water FP101). Para evaluar las características físicas del hábitat fluvial se apli-

Tabla 2. Valores medios de las variables físico-químicas del agua ( $\pm \mathrm{SE} ; n=6)$ y resultado de los análisis de la varianza (ANOVA) realizados. Letras diferentes señalan diferencias significativas entre los grupos establecidos por la prueba de Tukey $(\alpha=0.05)$. $\mathrm{PRS}=$ fósforo reactivo soluble. NID = nitrógeno inorgánico disuelto. Mean values $( \pm S E ; \mathrm{n}=6)$ of physicochemical variables and Analysis of Variance (ANOVA) results. Different uppercase denote significant differences as indicated by post-hoc Tukey test $(\alpha=0.05)$. $P R S=$ soluble reactive phosphorus. $N I D=$ dissolved inorganic nitrogen.

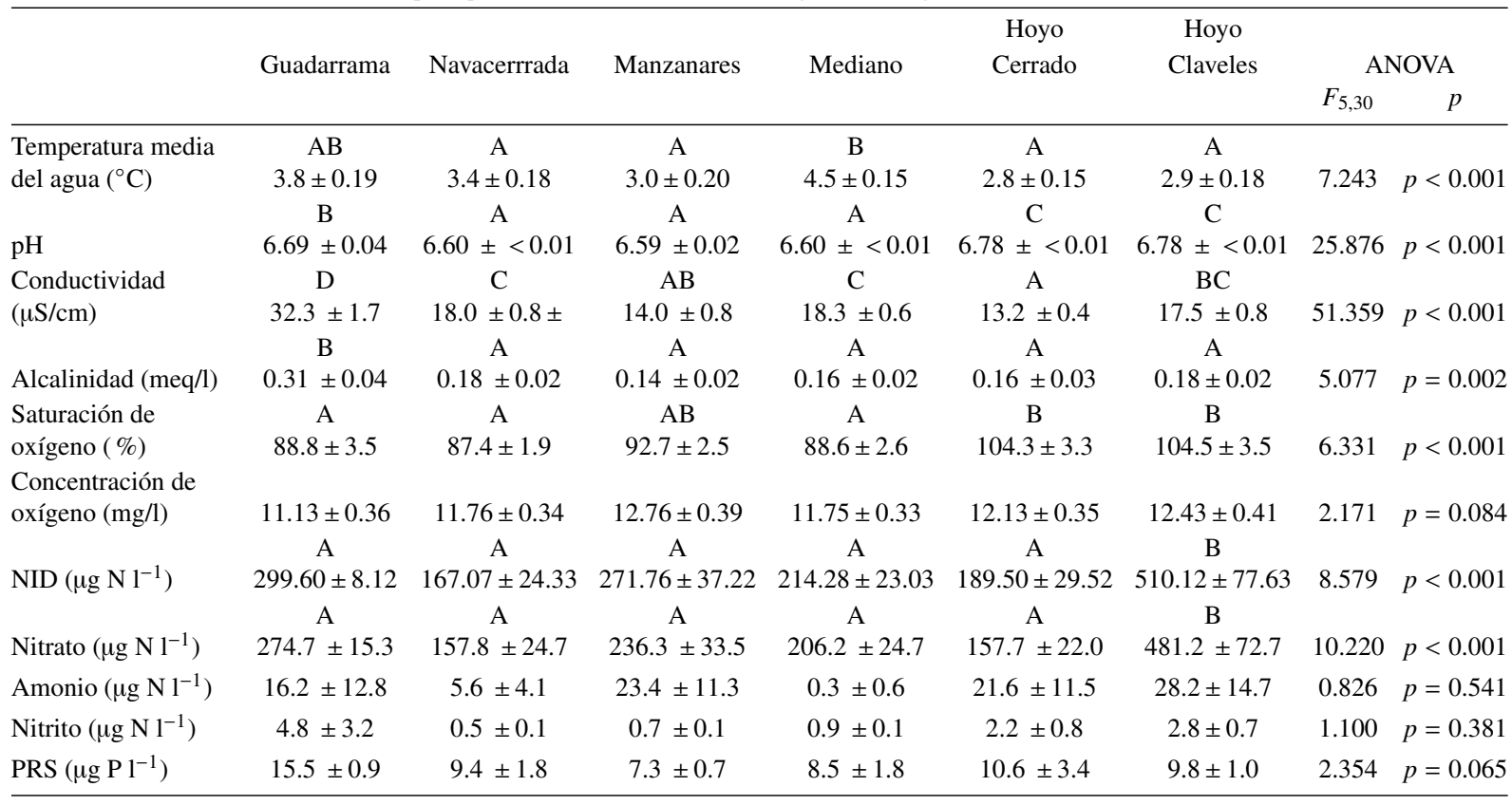


có el índice IHF (Pardo et al., 2002) y se utilizó el QBR (Munné et al., 2003) para evaluar la integridad del bosque de ribera. La cobertura del dosel de la vegetación riparia se estimó mediante un densiómetro esférico (Lemmon, 1956).

\section{Experimentos de descomposición con bolsas de hojarasca}

Las hojas de aliso (Alnus glutinosa (L.) Gaertner) utilizadas en los experimentos se recogieron justo después de la abscisión en otoño de 2007 y se secaron al aire hasta la obtención de peso constante. Posteriormente se pesaron porciones de $5.0 \pm 0.25 \mathrm{~g}$ que fueron humedecidas con un vaporizador e introducidas en bolsas de $15 \times$ $20 \mathrm{~cm}$ de tamaño y $5 \mathrm{~mm}$ de luz de malla. En cada arroyo, a lo largo de un tramo de $50 \mathrm{~m}$, se introdujeron 25 bolsas de hojarasca atadas con cuerdas de nailon a barras de hierro clavadas en el lecho. Para estimar la cantidad de masa perdida por lixiviación, en cada río se sumergieron 5 bolsas adicionales durante 24 horas. Después de 7 días se recuperaron las primeras cinco bolsas $\left(t_{7}\right)$ y las restantes en fechas que más o menos corresponden a las pérdidas del 20 ( $t_{20}, 12-14$ días $), 35\left(t_{35}\right.$, 27-30 días), 50 ( $t_{50}, 39-41$ días $), 70\left(t_{70}, 46-48\right.$ días) y $85 \%\left(t_{85}, 53-56\right.$ días $)$ de la masa inicial, estimadas a partir de las tasas de descomposición exponencial $(k)$ calculadas a partir de datos anteriores de cada estación experimental. Tras la recuperación de cada juego de cinco bolsas, éstas eran introducidas individualmente en bolsas de plástico y transportadas en nevera $\left(4^{\circ} \mathrm{C}\right)$ al laboratorio, donde eran procesadas inmediatamente. El material de cada bolsa de hojarasca se enjuagó con agua del río filtrada y la fauna fue separada en un tamiz de $200 \mu \mathrm{m}$ y conservada en etanol al $70 \%$ para su posterior análisis. Los macroinvertebrados recogidos en $t_{50}$ (cuando se produce normalmente su máximo de abundancia, Hieber \& Gessner, 2002) fueron contados e identificados a nivel de familia y clasificados en grupos funcionales de acuerdo con Merritt \& Cummins (1996) y Tachet et al. (2002). De cada bolsa se extrajo con un sacabocados un conjunto de cinco discos de hoja (12 mm de diámetro) para la determinación de la tasa de esporulación fúngica en $t_{20}$ (véase más adelante), cuando se espera el pico de producción de conidios (Pascoal \& Cássio 2004). El material restante fue, como en todas las fechas de muestreo, secado al horno $\left(70^{\circ} \mathrm{C}, 72 \mathrm{~h}\right) \mathrm{y}$ pesado. Una porción se utilizó para los análisis de nutrientes y el resto se quemó $\left(550^{\circ} \mathrm{C}, 4 \mathrm{~h}\right)$ para determinar el peso seco libre de cenizas (PSLC).

La porción para el análisis de nutrientes se molió en polvo fino con un molinillo analítico IKA-A10. El carbono (C) y el nitrógeno (N) se

Tabla 3. Tasas de descomposición de hojarasca, $k$ exponencial y $b$ lineal, de las hojas de aliso expresadas por tiempo (días, d) y calor acumulado (grados-día, dd). PSLC-Peso seco libre de cenizas. Exponential (k) and linear (b) leaf litter breakdown rates of alder leaves in terms of time (days, $d$ ) and accumulated heat (degree days, dd). PSLC-ash-free dry weight.

\begin{tabular}{|c|c|c|c|c|c|c|}
\hline & Guadarrama & Navacerrrada & Manzanares & Mediano & Hoyo Cerrado & Hoyo Claveles \\
\hline \multicolumn{7}{|c|}{ Modelo Exponencial } \\
\hline$k\left(\mathrm{dd}^{-1}\right)$ & 0.0148 & 0.0112 & 0.0128 & 0.0069 & 0.0214 & 0.0113 \\
\hline SE & 0.0028 & 0.0018 & 0.0022 & 0.0012 & 0.0024 & 0.0020 \\
\hline$R^{2}$ & 0.482 & 0.579 & 0.487 & 0.569 & 0.700 & 0.535 \\
\hline$k^{\prime}\left(\mathrm{d}^{-1}\right)$ & 0.053 & 0.037 & 0.035 & 0.029 & 0.053 & 0.031 \\
\hline SE & 0.011 & 0.007 & 0.035 & 0.005 & 0.007 & 0.006 \\
\hline$R^{2}$ & 0.508 & 0.599 & 0.549 & 0.584 & 0.746 & 0.552 \\
\hline \multicolumn{7}{|l|}{ Modelo Lineal } \\
\hline$b\left(\%\right.$ PSLC $\left.\cdot \mathrm{dd}^{-1}\right)$ & 0.432 & 0.482 & 0.496 & 0.302 & 0.639 & 0.496 \\
\hline $\mathrm{SE}$ & 0.040 & 0.039 & 0.496 & 0.027 & 0.053 & 0.048 \\
\hline$R^{2}$ & 0.827 & 0.844 & 0.768 & 0.833 & 0.842 & 0.796 \\
\hline$b^{\prime}\left(\%\right.$ PSLC $\left.\cdot \mathrm{d}^{-1}\right)$ & 1.62 & 1.62 & 1.41 & 1.31 & 1.65 & 1.38 \\
\hline $\mathrm{SE}$ & 0.14 & 0.13 & 0.15 & 0.11 & 0.13 & 0.13 \\
\hline$R^{2}$ & 0.810 & 0.851 & 0.803 & 0.823 & 0.842 & 0.794 \\
\hline
\end{tabular}


determinaron con un analizador elemental Perkin Elmer serie II CHNS/S (Perkin Elmer, Norwalk, Connecticut). El fósforo (P) se determinó espectrofotométricamente después de una digestión ácida mixta (método del azul de molibdeno, Allen et al., 1974). Los resultados se expresaron como \% de C, \% de $\mathrm{N}$ y \% de $\mathrm{P}$ del peso seco de hojarasca, y a lo largo del tiempo de descomposición como un porcentaje de los niveles de nutrientes iniciales en las hojas.

\section{La esporulación de los hifomicetos acuáticos}

Los discos de hojas extraídos de las bolsas de $t_{20}$ se incubaron en matraces Erlenmeyer de $100 \mathrm{ml}$ con $25 \mathrm{ml}$ de agua del río filtrada (Whatman $\mathrm{GF} / \mathrm{F}$ ) en un agitador (60 rpm) durante 48 horas a $10^{\circ} \mathrm{C}$. Las suspensiones de conidios resultantes se transfirieron a tubos de centrífuga de $50 \mathrm{ml}$ enjuagados dos veces con $2 \mathrm{ml}$ de agua destilada, se fijaron con $2 \mathrm{ml}$ de formalina al $37 \%$ y se tiñeron con azul de tripán en ácido láctico $(0.05 \%)$. Para la identificación y recuento de conidios se filtró una alícuota de la suspensión (Millipore SMWP 5- $\mu$ m de tamaño de poro). Los conidios fueron identificados y contados bajo un microscopio (Leica) a 250×. El peso seco de los discos de hojarasca se determinó como se ha descrito anteriormente. Las tasas de esporulación se expresaron como número de conidios producidos por mg de hoja de peso seco libre de cenizas por día ( $\mathrm{mg}^{-1}$ PSLC día $\left.^{-1}\right)$.

\section{Análisis de los datos}

Las diferencias en las variables físico-químicas entre los ríos se comprobaron mediante análisis de la varianza de una vía (ANOVA). Tras corregir la masa inicial de hojarasca para el lixiviado, las tasas de descomposición se estimaron mediante regresión lineal tanto para los datos originales (modelo lineal negativo $M_{t}=M_{0}-b * t$, donde $M_{0}$ es el PSLC inicial tras el lixiviado, $M_{t}$ es el PSLC remanente en el tiempo $t, \mathrm{y} b$ es la tasa de descomposición lineal) como para los datos transformados por logaritmo neperiano (modelo exponencial negativo $M_{t}=M_{0} * e^{-k t}$, donde $k$ es la tasa de descomposición exponencial) a fin de comparar ambos modelos. Para descartar el efecto de la variabilidad de la temperatura del agua entre ríos, las tasas de descomposición se calcularon también reemplazando el tiempo $(t)$ por la suma de las temperaturas medias diarias hasta el momento del muestreo (grados-día, dd). Las diferencias entre las tasas de descomposición se evaluaron mediante análisis de covarianza (ANCOVA, prueba de homogeneidad de pendientes) usando días y grados-día como covariable y río como factor. Las diferencias entre ríos para todas las demás variables estudiadas se evaluaron mediante ANOVAs de una vía, tras las que se realizaron pruebas de Tukey para buscar grupos homogéneos (Zar, 2010). Cuando los datos no cumplían los supuestos de normalidad se transformaron mediante arcoseno $(\sqrt{x})$ para cocientes y porcentajes, y logaritmo $\log (x+1)$ en otros casos. Las relaciones entre las variables estudiadas se evaluaron mediante el coeficiente de correlación de Spearman (rho) dado el bajo número de ríos. Todos los análisis estadísticos se realizaron con IBM SPPS Statistics 20 (IBM-SPSS).

\section{RESULTADOS}

\section{Características físicas y químicas del agua de los ríos}

Se trata de aguas frías con un bajísimo contenido en sales disueltas (conductividad eléctrica máxima de $32.3 \pm 1.7 \mu \mathrm{S} / \mathrm{cm}$ ), baja alcalinidad (máxima $0.31 \pm 0.04 \mathrm{meq} / \mathrm{l})$ y $\mathrm{pH}$ ligeramente ácido (entre 6.6-6.7) (Tabla 2). La variación espacial en estas características es pequeña, aunque estadísticamente significativa. La temperatura media del agua fue claramente más alta en el arroyo Mediano $\left(F_{5,30}=7.243, p<0.001\right)$, mientras que la alcalinidad y conductividad eléctrica fueron mayores en el río Guadarrama (Tabla 2). Estas aguas presentan un elevado porcentaje de oxígeno disuelto (próximo o ligeramente superior a la saturación) y una carga de nutrientes muy baja, típica de arroyos de cabecera oligotróficos. Los valores medios de PRS fueron muy bajos, variando de 7.3 en Manzanares a $15.5 \mu \mathrm{g} / \mathrm{l}$ en Guadarrama. La concentración de nitrógeno inorgánico disuelto 


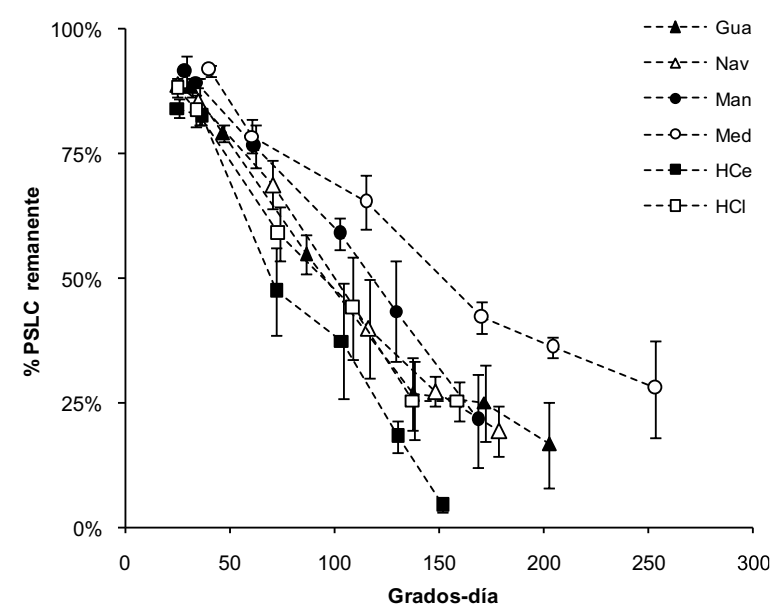

Figura 2. Porcentaje de hojarasca remanente en peso seco libre de cenizas (PSLC) en relación a los grados-día en la descomposición de las hojas de aliso en los ríos estudiados (promedio de 5 bolsas $\pm \mathrm{SE}$ ). Percentage of remaining ash-free dry mass (PSLC) in relation to degree days in the decomposition process of the alder leaves in the studied streams (mean of 5 bags $\pm S E$ ).

(NID) varió de 167.07 en Navacerrada a 510.12 $\mu \mathrm{g} / \mathrm{l}$ en Hoyo Claveles, mostrando este último una diferencia estadísticamente significativa respecto a los demás ríos (ANOVA, $F_{5,30}=8.579$, $p<0.001)$. El nitrato constituyó siempre el ión mayoritario de $\mathrm{N}$, mientras que el amonio supone una contribución irrelevante en prácticamente todos los ríos, encontrándose el nitrito en cantidades aún menores.

\section{Descomposición de la hojarasca y procesado de los nutrientes de las hojas}

La pérdida de masa de las hojas de aliso por lixiviado representó un 13-16\% de la masa inicial. En todos los ríos estudiados las dinámicas de descomposición de las hojas de aliso mostraron un mejor ajuste al modelo lineal que al exponencial, tanto en términos de tiempo (días, d) como de calor acumulado (grados-día, dd) (Tabla 3). Las tasas de descomposición por día se encontraron entre 1.31 y $1.65\left(\%\right.$ PSLC d $\left.\mathrm{d}^{-1}\right)$ y entre 0.302 y 0.639 cuando se calcularon frente a grados día (\%PSLC $\left.\mathrm{dd}^{-1}\right)$. No hubo diferencias estadísticamente significativas en las estimas de la tasa de descomposición por día (ANCOVA, $F_{5,168}=1.357, p=0.243$ ), pero sí apare- cían cuando la tasa de descomposición se corrigió para la temperatura calculándola por gradosdía (ANCOVA, $F_{5,168}=7.664, p<0.001$ ), siendo más rápida en Hoyo Cerrado y más lenta en el arroyo Mediano (Figura 2). La pérdida de masa de las hojas de aliso con el tiempo fue muy rápida, ya que antes de transcurridos 60 días se perdió más del $70 \%$ de la masa inicial, y en Hoyo Cerrado después de solo 30 días se perdió más del $50 \%$ de la masa inicial de hojarasca. La tasa de descomposición por día no se correlacionó significativamente con ningún factor abiótico de los estudiados (Tabla 4), mientras que la tasa de descomposición por grados-día sólo apareció significativamente correlacionada con la conductividad (rho $=-0.943, p=0.005)$. (Obviamos la relación con la temperatura por la dependencia entre temperatura con los grados día.) En ambos casos es de destacar la falta de correlación con factores relacionados con la abrasión de la hojarasca, como el caudal o la pendiente del tramo.

La calidad inicial del material fue la misma en todos los ríos, la composición relativa fue del $47.1 \% \pm 1.3$ de carbono (C), $2.48 \% \pm 0.14$ de nitrógeno $(\mathrm{N})$ y $0.08 \% \pm 0.003$ de fósforo $(\mathrm{P})$. $\mathrm{El}$ porcentaje de $\mathrm{C}$ en la hojarasca de aliso varió poco durante todo el proceso de descomposición (coeficiente de variación $(\mathrm{CV})$ promedio para todos los ríos $<5 \%$ ). Sin embargo, el contenido de $\mathrm{N}$ inicial aumentó durante los primeros días y al final de la descomposición en todos los ríos excepto en Hoyo Cerrado (que presentaba la tasa máxima de descomposición y los mínimos valores de nitrato) y en Hoyo Claveles (con los máximos valores de nitratos en el agua), por lo que, finalmente, las hojas de aliso en la masa de hojarasca ganaron $\mathrm{N}$ respecto al $\mathrm{C}$ (CV medio de $\mathrm{N} \approx 10 \%$ ) (Fig. 3a y Fig. 3c). En contraste, el contenido relativo de $\mathrm{P}$ experimentó una disminución inicial (tras 7 días) en Hoyo Cerrado y Manzanares, aumentando ligeramente en el resto, con fuertes variaciones después (Fig. 3b y Fig. 3d). En ningún caso se encontraron diferencias significativas en la proporción de $\mathrm{C}, \mathrm{N}$ o $\mathrm{P}$ entre los ríos ( $\% \mathrm{C}: F_{5,30}=1.198$, $p=0.335, \% \mathrm{~N}: F_{5,30}=0.227, p=0.335$ y $\% \mathrm{P}$ : $\left.F_{5,30}=0.750, p=0.893\right)$. La tasa de descomposición no se relacionó con la concentración de 
nutrientes en las hojas ni con la proporción de nutrientes (Tabla 4).

\section{Comunidades asociadas a la descomposición de la hojarasca}

\section{Diversidad de hifomicetos acuáticos y tasas de} esporulación

Se identificaron un total de 24 taxones de hifomicetos acuáticos esporulantes en las muestras $t_{20}$ recogidas a los 12-14 días de incubación (Tabla 5). La riqueza media de especies por estación fue muy baja, encontrando entre 9 y 13 especies en todos los ríos excepto en el Guadarrama, que registró un máximo de 18 . La riqueza media de taxones por bolsa no presentó diferencias estadísticamente significaticas $\left(F_{5,23}=2.209\right.$, $p=0.088$ ). Solo 6 taxones aparecieron en todos los ríos: Alatospora acuminata "subulada", Flagellospora curvula, Heliscus lugdunensis, Lemonniera alabamensis, Taeniospora gracilis var. enecta y Tetrachaetum elegans. Las especies claramente dominantes $(>50 \%$ de las tasas totales de esporulación) fueron Flagellospora curvula en Manzanares y Mediano, Heliscus lugdunensis en Navacerrada y Taeniospora gracilis var. enecta en Hoyo Claveles. En Guadarrama
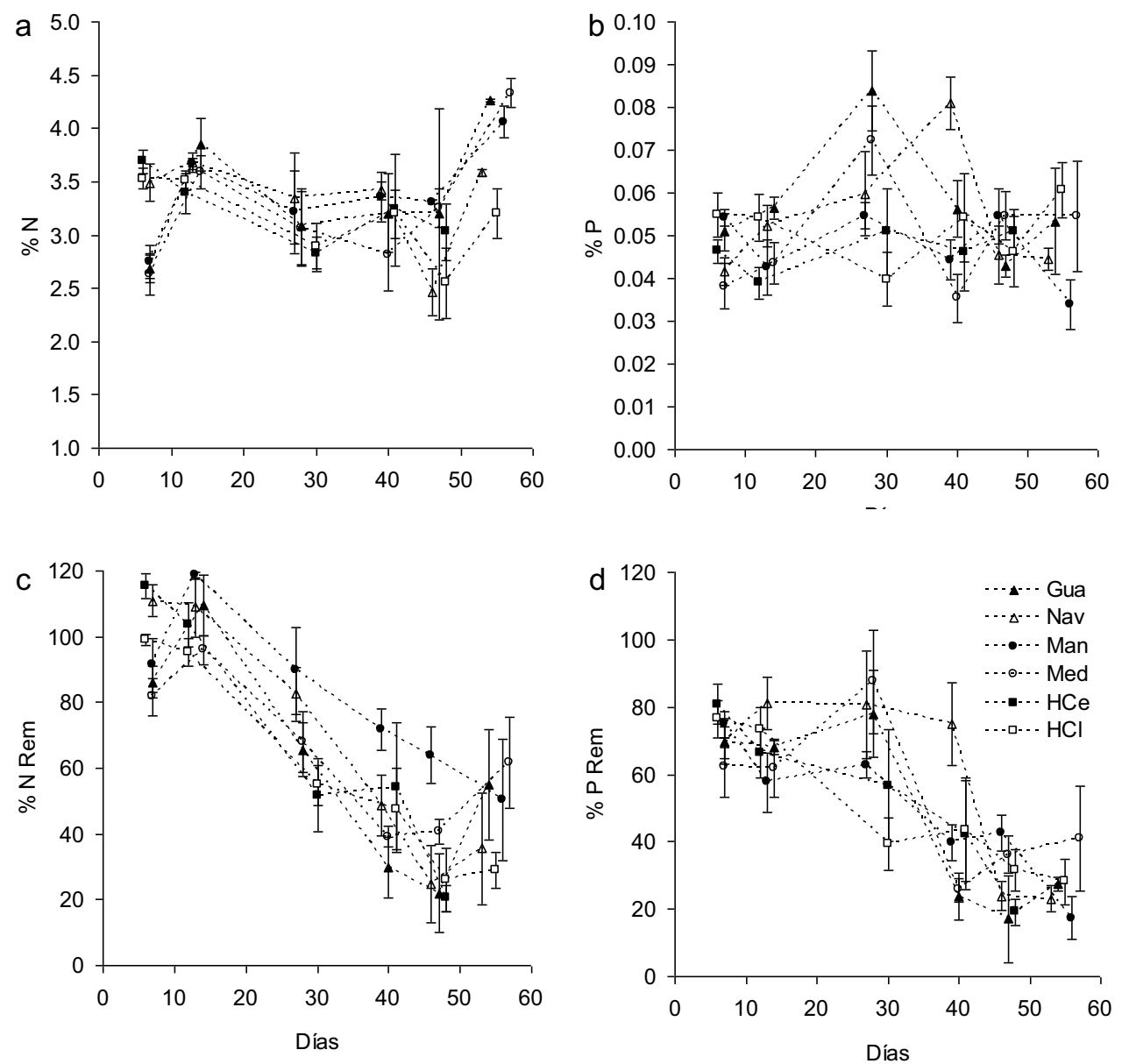

Figura 3. Contenido en nitrógeno (N) (a) y fósforo (P) (b) como porcentaje de la masa seca de hojarasca de aliso, y contenido en $\mathrm{N}$ (c) y fósforo $(\mathrm{P})(\mathrm{d})$ expresado como el porcentaje de los valores iniciales de $\mathrm{N}$ y de $\mathrm{P}$ respectivamente (media de 5 repeticiones $\pm \mathrm{SE})$. Nitrogen $(N)(a)$ and phosphorus $(P)(b)$ content expressed as percentage of alder litter dry mass, and $N(c)$ and $P(d)$ content expressed as the percentage of the initial $N$ and $P$ values, respectively (mean of 5 replicates $\pm S E$ ). 
y Hoyo Cerrado se observó un reparto más equitativo, con 4 especies codominantes (>5\% de las tasas totales de esporulación) en Guadarrama, Flagellospora curvula, Lemonniera alabamensis, Culidospora aquatica y Articulospora tetracladia, y 6 especies en Hoyo Cerrado, Flagellospora curvula, Taeniospora gracilis var. enecta, Heliscus lugdunensis, Tetrachaetum elegans, Anguillospora longissima y Alatospora acuminata "subulada".

Las tasas de esporulación variaron entre $3.27 \pm 1.77$ y $342.01 \pm 139.25$ conidios por $\mathrm{mg}$ PSLC y día, difiriendo significativamente entre los arroyos $\left(F_{5,23}=4.569, p<0.005\right)$. Los máximos valores se observaron en Guadarrama y Me- diano, que también presentaban una mayor temperatura del agua y riqueza de especies (Tablas 2 y 5), diferenciándose claramente del resto de los ríos, que mostraban valores excepcionalmente bajos a pesar de tener altas tasas de descomposición de hojarasca (Fig. 4a y 4b).

La tasa de esporulación se correlacionó con la temperatura media del agua (rho $=0.886$, $p=0.019)$ y la conductividad eléctrica (CE) (rho $=0.826, p=0.042$ ), pero no con la alcalinidad ni con ninguno de los nutrientes. La riqueza de HA no se correlacionó con ninguna de estas variables. No se encontró una relación significativa entre las tasas de esporulación de hifomicetos acuáticos y los indicadores de calidad

Tabla 4. Coeficientes de correlación de rango de Spearman entre las tasas de descomposición, $b$ en grados día y $b^{\prime}$ en días, de las hojas de aliso y las variables físico-químicas y biológicas. Spearman's rank correlation coefficients between degree day (b) and day $\left(b^{\prime}\right)$ leaf litter breakdown rates of alder leaves and physico-chemical and biological variables.

\begin{tabular}{|c|c|c|c|c|}
\hline & \multicolumn{2}{|c|}{$b^{\prime}\left(\%\right.$ PSLC $\left.\cdot \mathrm{d}^{-1}\right)$} & \multicolumn{2}{|c|}{$b\left(\%\right.$ PSLC $\left.\cdot \mathrm{dd}^{-1}\right)$} \\
\hline & rho & $p$ & rho & $p$ \\
\hline Caudal $\left(1 \mathrm{~s}^{-1}\right)$ & -0.203 & 0.700 & -0.029 & 0.957 \\
\hline Profundidad (cm) & -0.522 & 0.288 & -0.429 & 0.397 \\
\hline Pendiente media ( $\%$ ) & 0.580 & 0.228 & 0.029 & 0.957 \\
\hline Cobertura riparia & -0.203 & 0.700 & -0.429 & 0.397 \\
\hline IHF & -0.290 & 0.577 & -0.257 & 0.623 \\
\hline QBR & -0.088 & 0.868 & 0.638 & 0.173 \\
\hline $\mathrm{T}^{\mathrm{a}}$ media del agua $\left({ }^{\circ} \mathrm{C}\right)$ & -0.464 & 0.354 & -0.943 & 0.005 \\
\hline $\operatorname{PRS}\left(\mu g \mathrm{P} 1^{-1}\right)$ & 0.580 & 0.228 & 0.029 & 0.957 \\
\hline $\mathrm{NID}\left(\mu \mathrm{gN} 1^{-1}\right)$ & -0.435 & 0.389 & -0.086 & 0.872 \\
\hline Amonio $\left(\mu \mathrm{gN} 1^{-1}\right)$ & 0.232 & 0.658 & 0.771 & 0.072 \\
\hline Nitrito $\left(\mu \mathrm{gN} 1^{-1}\right)$ & 0.058 & 0.913 & -0.086 & 0.872 \\
\hline Nitrato $\left(\mu \mathrm{gN} 1^{-1}\right)$ & -0.435 & 0.389 & -0.086 & 0.872 \\
\hline $\mathrm{pH}$ & 0.313 & 0.545 & 0.265 & 0.612 \\
\hline Alcalinidad (meq/l) & -0.029 & 0.957 & -0.543 & 0.266 \\
\hline Conductividad $(\mu \mathrm{S} / \mathrm{cm})$ & -0.319 & 0.538 & -0.943 & 0.005 \\
\hline Concentración de oxígeno (mg/l) & -0.087 & 0.870 & 0.771 & 0.072 \\
\hline Saturación de oxígeno (\%) & 0.029 & 0.957 & 0.657 & 0.156 \\
\hline$\%$ de Carbono & 0.029 & 0.957 & -0.600 & 0.208 \\
\hline$\%$ de Nitrógeno & 0.406 & 0.425 & 0.200 & 0.704 \\
\hline$\%$ de Fósforo & -0.058 & 0.913 & -0.657 & 0.156 \\
\hline Cociente $\mathrm{C}: \mathrm{N}$ & -0.087 & 0.870 & -0.371 & 0.468 \\
\hline Cociente N:P & -0.116 & 0.827 & 0.600 & 0.208 \\
\hline Tasa de esporulación (conidios $\mathrm{mg}^{-1}$ PSLC $\mathrm{d}^{-1}$ ) & -0.348 & 0.499 & -0.771 & 0.072 \\
\hline Riqueza hifomicetos acuáticos & 0.145 & 0.784 & -0.429 & 0.397 \\
\hline $\mathrm{N}^{\mathrm{o}}$ de macroinvertebrados & -0.406 & 0.425 & 0.029 & 0.957 \\
\hline Riqueza (familias macroinvertebrados) & -0.725 & 0.103 & 0.143 & 0.787 \\
\hline $\mathrm{N}^{\mathrm{o}}$ de desmenuzadores & -0.232 & 0.658 & 0.371 & 0.468 \\
\hline
\end{tabular}


de la hojarasca (C:N y N:P), ni con las tasas de descomposición (Tabla 4).

\section{Comunidades de macroinvertebrados}

El número total de familias de macroinvertebrados encontrados en las bolsas de hojarasca en $t_{50}$ (después de 5-6 semanas de incubación) fue de 27. Hoyo Claveles y Manzanares presentaron una mayor riqueza total de familias (18 y 17 respectivamente) que Mediano y Navacerrada (14 y 13 respectivamente), con los valores inferiores en Hoyo Cerrado y Guadarrama (11 y 9) (ver Tabla 6). La riqueza media de familias por bolsa difirió significativamente entre los ríos $\left(F_{5,24}=2.971\right.$, $p=0.032$ ), aunque no se diferenciaron grupos netos sino la gradación comentada anteriormente entre los dos extremos. Los desmenuzadores estuvieron representados por un total de 9 familias $\mathrm{y}$ fueron el grupo funcional dominante de la comunidad de macroinvertebrados en todos los ríos excepto en Navacerrada. La riqueza de familias de desmenuzadores fue elevada (con 7 familias en la mayoría de los ríos) con la excepción de Navacerrada y Guadarrama (con 5 y 4 familias respectivamente), pero la riqueza de desmenuzadores por bolsa no difirió significativamente entre los ríos $\left(F_{5,24}=1.124, p=0.374\right)$.

La densidad de invertebrados por bolsa osciló en un rango muy estrecho (promedio 14.439.2 individuos) (Fig. 4c), aunque con diferencias significativas entre los ríos $\left(F_{5,24}=3.041\right.$, $p=0.029)$. La densidad total de invertebrados fue mayor en Hoyo Claveles, Manzanares y Guadarrama que en Navacerrada, Mediano y Hoyo Cerrado (Fig. 4c). La densidad relativa de desmenuzadores estaba comprendida entre el 30 y $91 \%$ de los macroinvertebrados totales, siendo llamativamente elevada en Hoyo Cerrado, Mediano y Manzanares (91.6, 72.3 y $69.9 \%$ respectivamente), muy alta en Hoyo Claveles y Guadarrama (62.2 y $60.7 \%$ ) y, aunque muy inferior, alta en Navacerrada (30.0\%), donde los colectores-recolectores (principalmente de la familia Chironomidae), presentaban un alto porcentaje $(43.33 \%)$. La densidad de desmenuzadores por bolsa (promedio 5.4-24.4 individuos) no difirió significativamente entre los ríos $\left(F_{5,24}=2.041\right.$, $p=0.109)$. Los desmenuzadores estaban representados mayoritariamente por tricópteros, de las familias Limnephilidae (en todos los ríos) y, secundariamente, Sericostomatidae (en Hoyo Claveles, Manzanares y Mediano), junto con Lepidostomatidae (en Hoyo Claveles), acompañados de plecópteros, familias Nemouridae (dominante en el río Manzanares), Leuctridae y Capniidae (especialmente en el Mediano).

No se observó ninguna correlación significativa entre los coeficientes de las tasas de descomposición y la riqueza o la densidad de invertebrados o desmenuzadores en $t_{50}$ (Tabla 4). Sin embargo, cuando analizamos la hojarasca perdida en cada bolsa en $t_{50}$ con la densidad de las familias de desmenuzadores en la bolsa, sí se encontró una relación significativa entre la pérdida de hojarasca por día y la densidad de Limnephilidae (rho $=0.480, p=0.007$ ), aún mayor si expresamos esta tasa de descomposición de hojarasca en grados-día (rho $=0.529, p=0.003$ ). Los Nemouridae presentaron un relación negativa con la pérdida de hojarasca por día (rho $=-0.542$, $p=0.002$ ), pero no con la perdida por grado día (rho $=-0.250, p=0.182$ ). El resto de los grupos de desmenuzadores no se correlacionaron con la pérdida de hojarasca por día ni por grado día.

\section{DISCUSIÓN}

Las tasas de descomposición de la hojarasca de aliso en los arroyos de la Sierra de Guadarrama fueron en general muy altas, semejantes e incluso superiores a los valores aportados por otros autores en ríos no alterados de la Selva Negra (Hieber \& Gessner, 2002) que ellos mismos consideran como en el extremo superior de valores registrados hasta el momento. Dentro de la Península Ibérica son superiores a los obtenidos por otros autores, como Pozo et al. (1997) en arroyos atlánticos, López et al. (1997) en un arroyo gallego, Pérez et al. (2011) en un estudio comparado entre arroyos de cabecera atlánticos del País Vasco y mediterráneos catalanes. Son similares a las tasas más rápidas registradas por diversos autores, como Casas et al. (2011) en ríos mediterráneos de montaña y mediterráneos semiáridos, 
Menéndez et al (2011) en arroyos mediterráneos, o Flores et al (2013) en pequeños arroyos oligotróficos atlánticos de características hidroquímicas semejantes a las aquí estudiadas.

Las tasas de descomposición variaron entre los arroyos dentro de un estrecho rango. Las diferencias en las características ambientales de los arroyos o en la temperatura del agua, mineralización y nutrientes disueltos no ayudan a explicar esta variación. La descomposición de la hojarasca fue siempre más rápida en Hoyo Cerrado, el arroyo de aguas más frías, mientras que en el arroyo Mediano la descomposición fue siempre la más lenta, a pesar de ser las aguas menos frías. Por lo que, en contra de la premisa de que la temperatura mejora la actividad biológica, especialmente de los microorganismos descomponedores, y por tanto acelera la descomposición de la hojarasca, nuestros resultados no apoyan ningún efecto positivo de la temperatura sobre el procesado de las hojas, por lo que otros factores pueden estar enmascarando su efecto (Gonçalves et al., 2006; Casas et al., 2011; Pozo et al., 2011; Pérez et al., 2013). Estos autores sugieren que las diferencias podrían estar relacionadas con la eficiencia de los consumidores y propusieron que las diferencias biológicas anulaban el efecto de la temperatura. Además, las mayores tasas se re-

Tabla 5. Contribución de las especies de hifomicetos acuáticos (\%) a la producción total de conidios, después de la perdida de hojarasca del $20 \%\left(t_{20}\right)$ en los 6 ríos estudiados. El porcentaje se calculó a partir del número total de esporas obtenidas en las 5 bolsas de cada río. Los datos de los taxones codominantes (los que contribuyen $>5 \%$ a la esporulación total por río) se destacan en negrita. Letras diferentes señalan diferencias significativas entre los grupos establecidos por la prueba de Tukey $(\alpha=0.05)$. Contribution ( \%) of the aquatic hyphomycete species to the total production of conidia, after mass loss $\left(\mathrm{t}_{20}\right)$ in the six studied streams. Percentage were calculated from the total number of spores in each stream $(\mathrm{n}=5)$. Data of co-dominant taxa (those with $>5 \%$ of total sporulation per stream) are highlighted in bold. Different uppercase denote significant differences as indicated by post-hoc Tukey test ( $\alpha=0.05)$.

\begin{tabular}{|c|c|c|c|c|c|c|}
\hline Especies & Guadarrama & Navacerrada & Manzanares & Mediano & Hoyo Cerrado & Hoyo Claveles \\
\hline Alatospora acuminata "pulchelloides"* & & & 0.23 & & & \\
\hline Alatospora acuminata sensu neotipo* & 0.04 & & & & & \\
\hline Alatospora acuminata "subulata"* & 0.12 & 0.27 & 1.39 & 0.03 & 5.29 & 1.14 \\
\hline Alatospora pulchella & 0.01 & & & & 0.58 & \\
\hline Anguillospora longissima & 0.03 & & & & 9.45 & 2.30 \\
\hline Anguillospora rosea & & & & & & 0.41 \\
\hline Articulospora tetracladia & 6.93 & 0.48 & 0.50 & 0.40 & & 0.39 \\
\hline Clavariopsis aquatica & & & & & & 0.39 \\
\hline Culicidospora aquatica & 23.70 & 4.89 & 0.28 & 0.52 & & \\
\hline Flagellospora curvula & 31.81 & 7.77 & 61.51 & 85.36 & 28.41 & 2.34 \\
\hline Geniculospora grandis & & & & 0.03 & & \\
\hline Geniculospora inflata & 2.94 & & & 1.21 & 1.77 & \\
\hline Heliscella stellata & & & 0.50 & & & \\
\hline Heliscus lugdunensis & 4.15 & 64.70 & 6.07 & 0.24 & 18.75 & 14.57 \\
\hline Lemonniera alabamensis & 24.56 & 1.46 & 0.25 & 0.16 & 3.24 & 0.80 \\
\hline Lemonniera aquatica & 1.04 & 0.76 & 2.15 & 0.16 & & \\
\hline Lemonniera centrosphaera & & & & & 0.89 & \\
\hline Lemonniera terrestris & 0.02 & & & 1.10 & 0.58 & \\
\hline Taeniospora gracilis var.enecta & 1.66 & 19.43 & 22.63 & 0.62 & 21.29 & 77.28 \\
\hline Tetrachaetum elegans & 2.62 & 0.24 & 4.48 & 10.07 & 9.76 & 0.39 \\
\hline Tetracladium marchalianum & 0.02 & & & & & \\
\hline Tetracladium setigerum & 0.01 & & & & & \\
\hline Tricladium chaetocladium & 0.34 & & & 0.11 & & \\
\hline Tricladium splendens & $<0.001$ & & & & & \\
\hline Tasa de esporulación & $\mathrm{B}$ & $\mathrm{AB}$ & A & $\mathrm{B}$ & A & A \\
\hline (conidios $\mathrm{mg}^{-1}$ PSLC d $^{-1}$ ) & $342.01 \pm 139.25$ & $6.20 \pm 1.47$ & $7.17 \pm 3.83$ & $227.42 \pm 140.40$ & $3.27 \pm 1.77$ & $4.07 \pm 2.64$ \\
\hline Riqueza específica & 18 & 9 & 11 & 13 & 11 & 10 \\
\hline
\end{tabular}

* Formas de los taxones segregados (véase Pozo et al., 2011). 
gistraron en los arroyos situados a mayor altitud (1400-1380 m.s.n.m.) que drenan áreas pequeñas, tienen caudales bajos y fluctuantes y una mayor pendiente. La pendiente del cauce afecta a la velocidad del agua y al transporte de partículas, lo que contribuye a la abrasión física de las hojas y acelera su fragmentación (Paul et al. 2006). Sin embargo, ni el caudal ni la pendiente ayudaron a explicar la variación de las tasas de descomposición en nuestro caso, por lo que la importancia de la abrasión física en el procesado de la hojarasca parece trivial en comparación con los efectos de los agentes bióticos (Hieber \& Gessner 2002, Ferreira et al. 2006, Hladyz et al. 2009).

El contenido de nutrientes de la hojarasca detritíca puede variar a lo largo del proceso de descomposición de acuerdo con la disponibilidad de nutrientes disueltos en el agua de los ríos. El aumento en el contenido de nutrientes de la hojarasca en descomposición se atribuye a la absorción de nutrientes microbianos y la inmovilización desde la columna de agua (Chauvet, 1987; Gulis \& Suberkropp, 2003), por lo que el aumento de la calidad de las hojas se puede considerar como un indicador indirecto de la actividad microbiana (Pérez et al., 2012). En nuestro caso, a pesar de la alta calidad de las hojas de aliso y de la baja disponibilidad de nutrientes en estas aguas oligotróficas frías, durante la incubación se observó un enriquecimiento de las hojas en $\mathrm{N}$ respecto al $\mathrm{C}$ en todos los ríos excepto en Hoyo Cerrado, un efecto ya indicado por otros autores (por ejemplo Pascoal \& Cássio, 2004). El contenido de P-foliar durante la descomposición experimentó una leve reducción, lo que es común en condiciones oligotróficas (Pozo, 1993; Pérez et al., 2011; Pérez et al., 2013), recuperando finalmente los valores iniciales. Únicamente se observó enriquecimiento o inmovilización de fósforo en Guadarrama y Mediano durante los primeros días y al final de la descomposición. La inmovilización del P-foliar se produce generalmente en relación con una mayor disponibilidad de nutrientes en el agua (Pozo, 1993), como parece ocurrir en Guadarrama, que presenta el valor máximo de PRS.

Excepto en Hoyo Cerrado, hubo un aumento de la calidad del recurso detrítico durante la des-
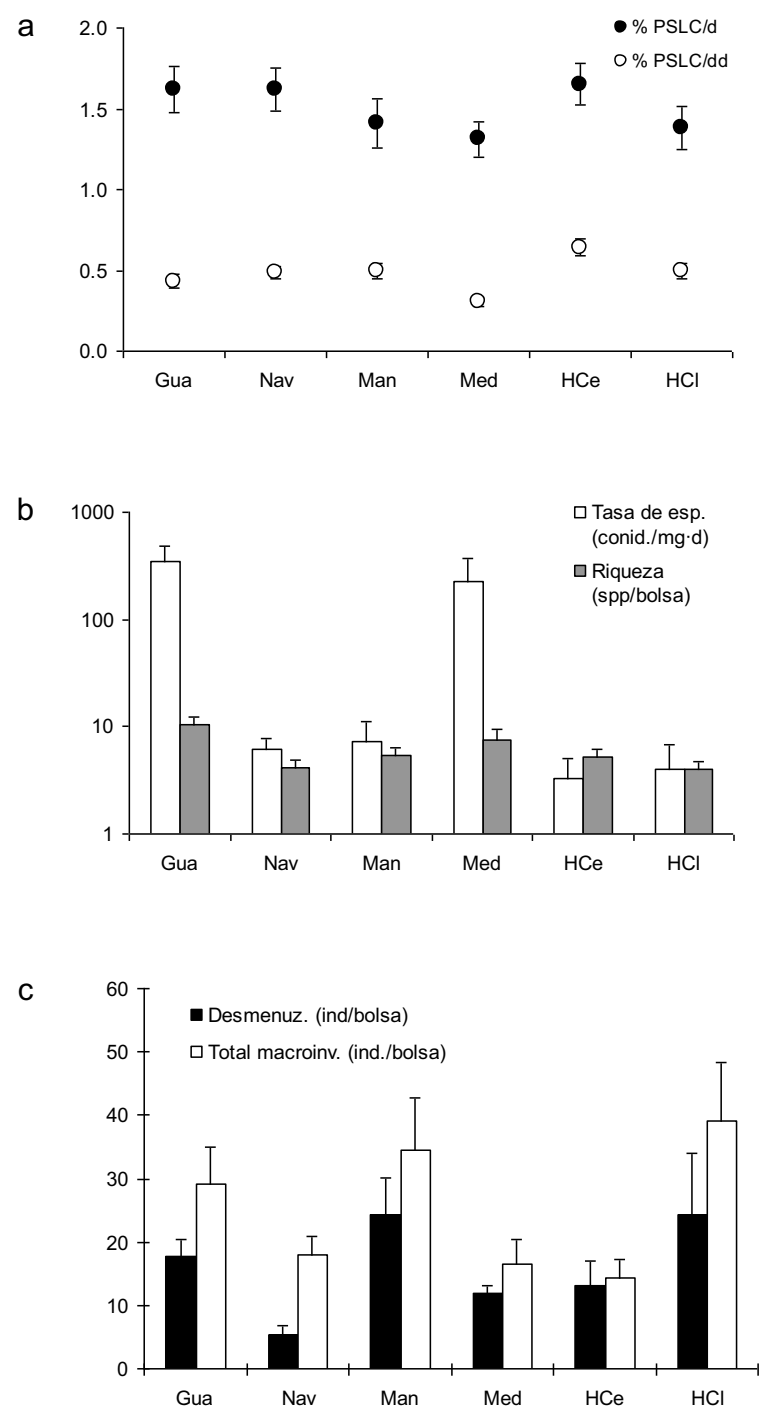

Figura 4. (a) Tasa de procesado lineal en días y grados-día (\% PSLC), (b) Tasa de esporulación de hifomicetos acuáticos (conidios/mg día ${ }^{-1}$ ) y riqueza específica (número de especies/bolsa) por río (eje $y$ a la izquierda en escala logarítmica, las barras indican el promedio $\pm \mathrm{SE}, n=5$ ), y (c) Densidad media de macroinvertebrados totales y desmenuzadores por río (promedio de 5 bolsas $\pm \mathrm{SE}$ ). (a) Linear processing rate in day and degree-day (\% PSLC), (b) mean hyphomycete sporulation rate and richness (number of species/litter bag) per stream (yaxis in logarithmic scale, bars $\pm S E ; \mathrm{n}=5$ ), and (c) mean total macroinvertebrate and shredder density (mean of 5 replicates $\pm S E)$.

composición por ganancia de $\mathrm{N}$ y $\mathrm{P}$ respecto al $\mathrm{C}$ (especialmente en Guadarrama y Mediano), a pesar de los bajos niveles de nutrientes del agua (rango de valores promedio PRS 7.3-15.5 $\mu \mathrm{g}$ P $\mathrm{l}^{-1}$ y Nitrato $157-481 \mu \mathrm{g} \mathrm{N}^{-1}$ ), lo que nos lleva 
a pensar en un mayor desarrollo e importancia relativa de la actividad de los hongos hifomicetos descomponedores en los ríos Guadarrama y Mediano y una escasísima importancia en Hoyo Cerrado, que sorprendentemente fue el que presentó las mayores tasas de descomposición. Coincidiendo con esta hipótesis la riqueza de hifomicetos acuáticos esporulantes fue muy baja, registrándose los valores más elevados en
Guadarrama y Mediano. Además se registró una fuerte dominancia de unas pocas especies, 4 en Guadarrama y sólo 2 en Mediano, y, contrariamente a lo esperado, el mayor reparto se observó en Hoyo Cerrado, con 6 especies codominantes entre las 11 encontradas en este arroyo. Aunque la riqueza de especies de hongos puede hacer aumentar la tasa de descomposición de hojarasca a través de efectos positivos sobre la calidad de los

Tabla 6. Abundancia total y grupos funcionales (GF) de las familias de macroinvertebrados encontradas en las cinco bolsas de hojarasca recogidas en cada uno de los seis ríos estudiados. $\mathrm{Shr}=$ desmenuzadores, $\mathrm{Col}=$ colectores, Gat $=$ recolectores, Filt $=$ filtradores, $\mathrm{Scr}=$ raspadores, Pre = depredadores. Total abundance of macroinvertebrate taxa and functional feeding groups found in five litter bags collected in each of the six studied streams. Shr $=$ shredder, Col $=$ collector, Gat $=$ collector-gatherer. Filt $=$ collectorfilterer, Scr = scraper, Pre = predator.

\begin{tabular}{|c|c|c|c|c|c|c|c|c|}
\hline GF & Orden & Familia & Guadarrama & Navacerrada & Manzanares & Mediano & Hoyo Cerrado & Hoyo Claveles \\
\hline Shr & Trichoptera & Limnephilidae & 55 & 10 & 12 & 21 & 38 & 45 \\
\hline Shr & Plecoptera & Leuctridae & 26 & 6 & 20 & 2 & 3 & 10 \\
\hline Shr & Plecoptera & Nemouridae & & & 56 & 1 & 11 & 2 \\
\hline Shr & Trichoptera & Sericostomatidae & 1 & 8 & 27 & 16 & 9 & 35 \\
\hline Shr & Trichoptera & Lepidostomatidae & & 1 & 4 & 2 & & 28 \\
\hline Shr & Plecoptera & Capniidae & 6 & 2 & 1 & 15 & & 1 \\
\hline Shr & Diptera & Tipulidae & & & 1 & & 3 & 1 \\
\hline Shr & Diptera & Limoniidae & & & & 3 & 1 & \\
\hline Shr & Plecoptera & Taeniopterygidae & & & & & 1 & \\
\hline Col-Gat & Diptera & Chironomidae & 33 & 36 & 24 & 4 & & 29 \\
\hline Col-Gat & & Oligochaeta & & & & & 1 & \\
\hline Col-Gat & Ephemeropte & Leptophlebiidae & 16 & 3 & 2 & & & 14 \\
\hline Col-Gat & Diptera & Psychodidae & & & & 3 & & \\
\hline Col-Gat & Ephemeropte & Ephemerellidae & & & & 1 & & \\
\hline Col-Filt & Trichoptera & Hydropsychidae & & & 9 & & 3 & 3 \\
\hline Col-Filt & Diptera & Simuliidae & 3 & 6 & 9 & 9 & & 12 \\
\hline Col-Filt & Trichoptera & Philopotamidae & & & & & & 1 \\
\hline Scr & Coleoptera & Scirtidae & & 7 & & 3 & & 4 \\
\hline Scr & Trichoptera & Goeridae & & 1 & 1 & 2 & & 3 \\
\hline \multicolumn{3}{|c|}{ Col-Gat-Scr Ephemeroptera Baetidae } & 4 & & 1 & & 1 & \\
\hline \multicolumn{2}{|c|}{ Col-Gat-Scr Coleoptera } & Elmidae & & & & & & 1 \\
\hline \multicolumn{2}{|c|}{ Col-Gat-Scr Coleoptera } & Hydraenidae & & 1 & & & & \\
\hline Pre & Turbellaria & Planariidae & & 3 & & & & 3 \\
\hline Pre & Plecoptera & Perlidae & & & 1 & & & \\
\hline Pre & Diptera & Athericidae & & & 1 & & & 1 \\
\hline Pre & Trichoptera & Rhyacophilidae & & & 3 & & 1 & \\
\hline Pre & Plecoptera & Chloroperlidae & 1 & 6 & 1 & 1 & & 3 \\
\hline \multicolumn{3}{|c|}{ Número de individuos } & 145 & 90 & 173 & 83 & 72 & 196 \\
\hline \multicolumn{3}{|c|}{ Número de familias } & 9 & 13 & 17 & 14 & 11 & 18 \\
\hline \multicolumn{3}{|c|}{ Número de familias de desmenuzadores } & 4 & 5 & 7 & 7 & 7 & 7 \\
\hline \multicolumn{3}{|c|}{ Media familias por bolsa $( \pm S E)$} & $\mathrm{AB}$ & $\mathrm{AB}$ & $\mathrm{AB}$ & $\mathrm{AB}$ & A & A \\
\hline \multirow{2}{*}{\multicolumn{3}{|c|}{ Media familias de desmenuzadores por bolsa $( \pm \mathrm{SE})$}} & $5.6 \pm 0.60$ & $6.2 \pm 0.58$ & $6.8 \pm 1.39$ & $5.0 \pm 1.05$ & $4.2 \pm 0.74$ & $9.2 \pm 3.03$ \\
\hline & & & $3.0 \pm 0.00$ & $2.6 \pm 0.51$ & $3.4 \pm 0.87$ & $2.8 \pm 0.74$ & $3.0 \pm 0.45$ & $4.4 \pm 0.68$ \\
\hline
\end{tabular}


recursos para los desmenuzadores (Lecerf et al. 2005), tal relación no se observó en nuestro caso.

Las tasas medias y totales de esporulación fueron extraordinariamente bajas, a pesar de que el tiempo transcurrido desde la implantación hasta $t_{20}$ (de 12 a 14 días) parece coincidir con la fase de crecimiento de micelios en la hojarasca (Pozo et al. 1998) y es un período de alta producción de esporas fúngicas (Chauvet et al. 1997). Flores et al. (2013) encontraron la máxima tasa de esporulación a los 15 días de incubación en 3 tipos de ambientes en el arroyo Malbazar del Parque Natural de Aiako Harria, siendo las especies de hifomicetos dominantes semejantes a las nuestras y ligadas a arroyos de baja mineralización.

Las relaciones entre la actividad fúngica, la disponibilidad de nutrientes y la descomposición de las hojas en aguas pobres en nutrientes son probablemente complejas. En nuestro estudio, el promedio de $\mathrm{NO}_{3}-\mathrm{N}$ disuelto mostró un gradiente desde 157 a $481 \mu \mathrm{g} / \mathrm{l}$ y el promedio de SRP osciló desde 7.3 a $15.5 \mu \mathrm{g} / \mathrm{l}$. Estas concentraciones, aunque bajas, son suficientes para satisfacer las demandas fúngicas de $\mathrm{N}$ y $\mathrm{P}$ (Artigas et al. 2008) y provocar una respuesta tanto en las tasas de descomposición como en las tasas de esporulación (Grattan \& Suberkropp 2001), pero aunque no se observó que la disponibilidad de nutrientes acelerara la descomposición, la tasa de esporulación estaba positivamente relacionada con el PRS. Las mayores tasas de esporulación, aunque bajas, se observaron en Guadarrama y Mediano (los de aguas menos frías), claramente diferenciados del resto de los ríos, que presentaron valores muy inferiores y excepcionalmente bajos a pesar de que es en estos arroyos donde se encontraron las mayores tasas de descomposición de hojarasca. La tasa mínima de esporulación se registró en Hoyo Cerrado, que es el que claramente presenta la tasa más rápida de descomposición. Flores et al. (2013) tampoco encontraron una relación significativa entre la tasa de descomposición y la tasa de esporulación, lo que atribuyen a que la esporulación es una inversión de los hongos que no tendría una correlación sencilla con el crecimiento de las hifas ni con la degradación de la materia orgánica. Sin embargo, en nuestro caso la tasa de esporulación y la tasa de descomposi- ción parecen seguir un patrón inverso: las mayores tasas de esporulación se encuentran donde las tasas de descomposición fueron más bajas. Esto nos lleva a pensar en primer lugar en una escasa actividad fúngica, siendo otro tipo de factores o de organismos los que podrían tener más importancia en la degradación y consumo del material detrítico, lo que sugeriría una mayor importancia relativa de los invertebrados frente a los microorganismos en estas aguas frías y pobres en nutrientes. Además podría sugerir que las tasas de descomposición fueron demasiado rápidas para permitir una buena colonización y acondicionamiento de la hojarasca por parte de los hongos, por ejemplo en Hoyo Cerrado, y que los invertebrados desmenuzadores ya habrían consumido una importante cantidad del material detrítico en $t_{20}$, antes de que los hongos tuviesen tiempo suficiente para colonizar el sustrato y desarrollarse activamente. Si esta sugerencia es correcta, los invertebrados desmenuzadores deberían desempeñar un papel decisivo en la descomposición de las hojas, especialmente si la actividad fúngica es limitada (Pozo et al., 2011).

La riqueza y densidad de macroinvertebrados encontrados en las bolsas de hojarasca no fueron muy elevadas y oscilaron dentro de rangos estrechos, quizá debido al rápido procesado del material detrítico, ya que en $t_{50}$ quedaba muy poca hojarasca en las bolsas, y a la escasa heterogeneidad y variabilidad ambiental entre estos ríos bien conservados. Sin embargo, resulta llamativa la elevadísima proporción de desmenuzadores encontrados en las bolsas de hojarasca, en especial en Hoyo Cerrado, el río con las tasas más elevadas de descomposición. Parece ser que las bolsas de hojarasca no fueron utilizadas como refugio sino como recurso alimenticio de alta calidad que fue consumido rápidamente. Las hojas de aliso son muy nutritivas, por lo que en estos arroyos puede suponer una comida apetitosa en una despensa de detritos menos apetecible y nutritiva, y atraer así una cantidad desproporcionada de desmenuzadores (Hagen et al., 2006; Tiegs et al., 2008). Ésta podría ser la causa de las elevadas tasas de descomposición encontradas en los arroyos de la Sierra de Guadarrama. Sin embargo, con la excepción de Hoyo Cerrado, las dife- 
rencias entre ríos en la densidad de macroinvertebrados, en la densidad de desmenuzadores o en su abundancia relativa no se traducen en cambios significativos de las tasas de descomposición, como ya observaron Casas et al. (2000) y Niu \& Dudgeon, (2011). Por lo que, dada la importancia que este grupo funcional tiene en la descomposición de la hojarasca y que no hay otros factores que hayan dado lugar a una relación significativa con las tasas de descomposición obtenidas en el presente estudio, las pequeñas diferencias en la composición, biomasa, tamaño y estrategias específicas de alimentación de los desmenuzadores podrían explicar la variabilidad observada en la descomposición de la masa foliar.

Entre los desmenuzadores encontramos exclusivamente larvas de insectos, con una ausencia completa de crustáceos y caracoles ligada a las características de estos arroyos montañosos silíceos que presentan aguas frías, blandas y bajo contenido en calcio. Los desmenuzadores encontrados fueron básicamente tricópteros (Limnephilidae, Sericostomatidae y Lepidostomatidae) y plecópteros (Leuctridae, Nemouridae, Capniidae y Taeniopterygidae), con una menor abundancia de dípteros (Tipulidae y Limoniidae). Esto concuerda con los resultados de otros autores que han observado que las aguas frías pueden favorecer el rendimiento de algunos desmenuzadores como plecópteros y tricópteros, ya que presentan varias adaptaciones a los regímenes térmicos fríos (Danks, 2007) que podrían estar relacionadas con las eficiencias de consumo (Gonçalves et al., 2006). Pero no sólo la densidad de desmenuzadores es importante sino también su biomasa y su tamaño corporal. Los grandes desmenuzadores como los tricópteros y dípteros juegan un papel decisivo en el consumo del material detrítico, ya que sus necesidades metabólicas están relacionadas con su tamaño corporal (Reiss et al., 2011; Dossena et al., 2012). Además, tanto Tipula como muchas larvas de tricópteros limnefílidos comen todas las partes de la hoja, incluyendo el mesófilo y la venación (Ward \& Woods, 1986), y son capaces de digerir y asimilar los polisacáridos de la pared celular de las plantas. Es decir, mastican, digieren y asimilan la hoja entera, siendo menos dependientes de la fase de acondi- cionamiento, pero a costa de un alto gasto energético (Barlöcher, 1983). Además, algunos tricópteros como Sericostoma son capaces de alimentarse de forma efectiva incluso de acículas de pino sin apenas acondicionar (Campos \& González, 2009). Todo esto les confiere enormes ventajas frente a otros grandes desmenuzadores que no aparecen en nuestros ríos, como por ejemplo los gammáridos, que son incapaces de procesar un material detrítico poco acondicionado por los hifomicetos.

De esta forma, los grandes tricópteros y dípteros desmenuzadores que no necesitan un material bien acondicionado, y que se han encontrado profusamente dentro de las bolsas de hojarasca, pudieron ser los responsables de las elevadas tasas de descomposición encontradas en los ríos de referencia de la Sierra de Guadarrama, en los que desempeñarían un papel clave en el procesamiento de la hojarasca frente a la limitada actividad fúngica (indicado por las bajas tasas de esporulación), y ayudarían a explicar las tasas de descomposición más rápidas en los arroyos más fríos donde los consumidores (estos desmenuzadores), más que los descomponedores (los hifomicetos), parecieron ejercer un control decisivo en la velocidad del proceso. Lo contrario nos ocurrió en los arroyos menos fríos (Mediano y Guadarra$\mathrm{ma}$ ), donde los descomponedores podrían desempeñar un papel algo más importante frente a los consumidores.

Por último, resaltar que las tasas de descomposición no sólo fueron altas sino también relativamente homogéneas entre los distintos ríos; sólo aparecen diferencias significativas cuando acentuamos estas diferencias al corregir las tasas por la temperatura y expresarlas en grados-día. Además, convendría profundizar en el conocimiento de los organismos que participan en el proceso de descomposición en estos sistemas y comprobar si efectivamente, tal y como podemos suponer a partir de estos resultados, los macroinvertebrados consumidores (desmenuzadores) tienen una mayor relevancia en el procesado de los restos vegetales en los ríos de montaña con aguas más frías, mientras que en los ríos más cálidos sería mayor la importancia de los descomponedores (hifomicetos acuáticos). 


\section{AGRADECIMIENTOS}

Queremos agradecer a la dirección y personal del Parque Regional de la Cuenca Alta del Manzanares y del Parque Natural de Peñalara las facilidades de acceso y permisos de muestreo. También expresar nuestro agradecimiento a Fernando Rodríguez, Carolina Rozas y Roberto Velilla por la ayuda prestada en el trabajo de campo y laboratorio. Este estudio ha sido financiado por el Ministerio de Educación y Ciencia (CGL2007-66664C04).

\section{BIBLIOGRAFÍA}

ALLEN, S. E., J. A. GRIMSHAW, J. A. PARKINSON \& C. QUARMBY. 1974. Chemical analysis and Ecological Materials. Blackwell, Oxford.

APHA. 2005. Standard Methods for the Examination of Water and Wastewater. American Public Health Association, Washington.

ARTIGAS, J., A. M. ROMANÍ \& S. SABATER. 2008. Effect of Nutrients on the Sporulation and Diversity of Aquatic Hyphomycetes on Submerged Substrata in a Mediterranean Stream. Aquatic Botany 88: 32-38.

BARLÖCHER, F. 1983. Seasonal Variation of Standing Crop and Digestibility of CPOM in a Swiss Jura Stream. Ecology, 64: 1266-1272

BOE 229/2008 (de 22 de septiembre de 2008) 15340 ORDEN ARM/2656/2008, de 10 de septiembre, por la que se aprueba la instrucción de planificación hidrológica.

BORDALLO, A. \& C. CASADO. 2012. Aproximación al establecimiento de una red de referencia para la implantación de la Directiva Marco del Agua en la Comunidad de Madrid. Limnetica, 31(1): 112.

CAMPOS, J. \& J. M. GONZÁLEZ. 2009 Sericostoma vittatum (Trichoptera) Larvae Are Able to Use Pine Litter as an Energy Source. International Review of Hydrobiology, 94(4): 472-483

CARLISLE, D. M. \& W. H. CLEMENT. 2005. Leaf Litter Breakdown, Microbial Respiration and Shredder Production in Metal-Polluted Streams. Freshwater Biology, 50: 380-390.

CASADO, C., L. LOSADA \& S. MOLLÁ. 2011. Efecto de las áreas de protección especial en la comunidad de macroinvertebrados de los ríos de la cuenca alta del Manzanares (Sierra de Guadarrama, Madrid). Limnetica, 30(1): 71-88.

CASAS, J. J. \& M. O. GESSNER. 1999. Leaf Litter Breakdown in a Mediterranean Stream Characterized by Travertine Precipitation. Freshwater Biology, 41: 781-793.

CASAS, J. J., C. ZAMORA-MUÑOZ, F. ARCHILA \& J. ALBA-TERCEDOR. 2000. The Effect of a Headwater Dam on the Use of Leaf Bags by Invertebrate Communities. Regulated Rivers: Research \& Management, 16: 577-591.

CASAS, J. J., M. O. GESSNER, P. H. LANGTON, D. CALLE, E. DESCALS \& M. J. SALINAS. 2006. Diversity of Patterns and Processes in Rivers of Eastern Andalusia. Limnetica, 25(1-2): 155-170.

CASAS, J. J., M. O. GESSNER, D. LÓPEZ \& E. DESCALS. 2011. Leaf-Litter Colonisation and Breakdown in Relation to Stream Typology: Insights from Mediterranean Low-Order Streams. Freshwater Biology, 56: 2594-2608.

CASAS, J. J., A. LARRAÑAGA, M. MENÉNDEZ, J. POZO, A. BASAGUREN, A. MARTÍNEZ, J. PÉREZ, J. M. GONZÁLEZ, S. MOLLÁ, C. CASADO, E. DESCALS, N. ROBLAS, J. A. LÓPEZ-GONZÁLEZ \& J. L. VALENZUELA. 2013. Leaf Litter Decomposition of Native and Introduced Tree Species of Contrasting Quality in Headwater Streams: How does the Regional Setting Matter? Science of the Total Environment, 458460: 197-208.

CHAUVET, E. 1987. Changes in the Chemical Composition of Alder, Poplar and Willow Leaves During Decomposition in a River. Hydrobiologia, 148: 35-44.

CHAUVET, E., E. FABRE, E. ELOSEGUI \& J. POZO. 1997. The Impact of Eucalypt on the LeafAssociated Aquatic Hyphomycetes in Spanish Streams. Canadian Journal of Botany, 75: 880888.

DANKS, H. V. 2007. How Aquatic Insects Live in Cold Climates. Canadian Entomologist, 139: 443471.

DANGER, A. R. \& B. J. ROBSON. 2004. The Effects of Land Use on Leaf-Litter Processing by Macroinvertebrates in an Australian Temperate Coastal Stream. Aquatic Sciences, 66: 296-304.

DOSSENA, M., G. YVON-DUROCHER, J. GREY, J. M. MONTOYA, D. M. PERKINS, M. TRIMMER. 2012. Warming Alters Community Size Structure 
and Ecosystem Functioning. Proceeding of the Toyal Society, 279: 3011-3019.

ELOSEGUI, A., A. BASAGUREN \& J. POZO. 2006. A Functional Approach to the Ecology of Atlantic Basque Streams. Limnetica, 25(1-2): 123-134.

FEIO, M. J., T. ALVES, M. BOAVIDA, A. MEDEIROS \& M. A. S. GRAÇA. 2010. Functional Indicators of Stream Health: A River-Basin Approach. Freshwater Biology, 55: 1050-1065.

FERREIRA, V., V. GULIS \& M. A. S. GRAÇA. 2006. Whole Stream Nitrate Addition Affects Litter Decomposition and Associated Fungi But Not Invertebrates. Oecologia, 149: 718-729.

FLORES, L., J. R. DÍEZ, A. LARRAÑAGA, C. PASCOAL \& A. ELOSEGI. 2013. Effects of Retention Site on Breakdown of Organic Matter in a Mountain Stream. Freshwater Biology, 58: 1267-1278.

GESSNER, M. O. \& E. CHAUVET. 2002. A Case for Using Litter Breakdown to Assess Functional Stream Integrity. Ecological Applications, 12: 498-510.

GONÇALVES, Jr. J. F., M. A. S. GRAÇA \& M. CALLISTO. 2006. Leaf Litter Breakdown in 3 Streams in Temperature, Mediterranean and Tropical Cerrado Climates. Journal of North American Benthological Society, 24: 344-355.

GRAÇA, M. A. S. 2001. The Role of Invertebrates on Leaf Litter Decomposition in Streams -A Review. International Review of Hydrobiology, 86: 383393.

GRAÇA, M. A. S. \& C. CANHOTO. 2006. Leaf Litter Processing in Low Order Streams Limnetica, 25(1-2): 1-10.

GRATTAN, R. M. \& K. SUBERKROPP. 2001. Effects of Nutrient Enrichment on Yellow Poplar Leaf Decomposition and Fungal Activity in Streams. Journal of the North American Benthological Society, 20: 33-43.

GULIS, V. \& K. SUBERKROPP. 2003. Effect of Inorganic Nutrients on Relative Contribution of Fungi and Bacteria to Carbon Flow from Submerged Decomposing Leaf Litter. Microbial Ecology, 45: 1119.

HALL, R. O., J. B. WALLACE \& S. L. EGGERT. 2000. Organic Matter Flow in Stream Food Webs with Reduced Detritical Resource Base. Ecology, 81: 3445-3463.

HAGEN, E. M., J. R. WEBSTER \& E. F. BENFIELD. 2006. Are Leaf Breakdown Rates Useful to Measure of Stream Integrity along an Agricultural Landuse Gradient? Journal of the North American Benthological Society, 25: 330-343.

HIEBER, M. \& M. GESSNER. 2002. Contribution of Stream Detritivores, Fungi, and Bacteria to Leaf Breakdown Based on Biomass Estimates. Ecology, 83(4): 1026-1038.

HLADYZ, S., M. O. GESSNER, P. S. GILLER, J. POZO \& G. WOODWARD. 2009. Resource Quality and Stoichiometric Constraints on Stream Ecosystem Functioning. Freshwater Biology, 54: 957-970.

KROM, M. D. 1980. Spectrophotometric Determination of Ammonia: A Study of a Modified Berthelot Reduction Using Salicylate and Dichloroisocyanurate. Analyst, 105: 305-316.

LECERF, A., M. DOBSON, Ch. DANG \& E. CHAUVET. 2005. Riparian Plant Species Loss Alters Trophic Dynamics in Detritus-Based Stream Ecosystems. Oecologia, 146: 432-442.

LECERF, A., P. USSEGLIO-OLATERA, J. Y. CHARCOSSET, D. LAMBRIGOT, B. BRACHT \& E. CHAUVET. 2006. Assessment of Functional Integrity of Eutrophic Streams Using Litter Breakdown and Benthic Macroinvertebrates. Archiv für Hydrobiologie, 165: 105-126.

LEMMON, P. E. 1956. A Spherical Densitometer for Estimating Forest Overstory Density. Forest Science, 2: 314-320.

LÓPEZ, E. S., N. FELPETO \& I. PARDO. 1997. Comparisons of Methods to Study the Processing of Alnus glutinosa and Eucalyptus globulus Leaves in a Forested Headwater Stream. Limnetica, 13(2): 13-18.

MARTÍNEZ, A., A. LARRAÑAGA, A. BASAGUREN, J. PÉREZ, C. MENDOZA-LERA \& J. POZO. 2013. Stream Regulation by Small Dam Affects Benthic Macroinvertebrate Communities: From Structural Changes to Functional Implications. Hydrobiologia, 711: 31-42.

MENÉNDEZ, M., M. MARTÍNEZ, O. HERNÁNDEZ \& F. A. COMÍN. 2001. Comparison of Leaf Decomposition in Two Mediterranean Rivers: A Large Eutrophic River and an Oligotrophic Stream (S Catalonia, NE Spain). International Review of Hydrobiology, 86(4-5): 475-486.

MENÉNDEZ, M., E. DESCALS, T. RIERA \& O. MOYA. 2011. Leaf Litter Breakdown in Mediterranean Streams: Effect of Disssolved Inorganic Nutrients. Hydrobiologia, 669: 143-155. 
MERRITT, R. W. \& K. W. CUMMINS. 1996. An Introduction to the Aquatic Insects of North America. Kendall/Hunt Publishing Company, Dubuque.

MONTEIRO, M .I. C., F. N. FERREIRA, N. M. M. DE OLIVEIRA \& A. K. ÁVILA. 2003. Simplified Version of the Sodium Salicylate Method for Analysis of Nitrate in Drinking Waters. Analytica Chimica Acta, 477: 125-129.

MUNNÉ, A., N. PRAT, C. SOLA, N. BONADA \& M. RIERADEVALL. 2003. A Simple Field Method for Assessing the Ecological Quality of Riparian Habitat in Rivers and Streams: QBR index. Aquatic Conservation: Marine and Freshwater Ecosystems, 13: 147-163.

NIU, S. Q. \& D. DUDGEON. 2011. The Influence of Flow and Seasonal upon Leaf' Litter Breakdown in Monsoonal Hong Kong Streams. Hydrobiologia, 663: 205-215.

PAUL, M. J., J. L. MEYER \& C.A. COUCH. 2006. Leaf Breakdown in Stream Differeng in Catchment Land Use. Freshswater Biology, 51: 1684-1695.

PARDO, I., M. ÁlVAREZ, J. CASAS, J. L. MORENO, S. VIVAS, N. BONADA, J. ALBATERCEDOR, P. JÁIMEZ-CUÉLLAR, G. MOYÀ, N. PRAT, S. ROBLES, M. L. SUÁREZ, M. TORO \& M. R. VIDAL-ABARCA. 2002. El hábitat de los ríos mediterráneos. Diseño de un índice de diversidad de hábitat. Limnetica, 21: 115-133.

PASCOAL, C. \& F. CÁSSIO. 2004. Contribution of Fungi and Bacteria to Leaf Litter Decomposition in a Polluted River. Applied and Environmental Microbiology, 70: 5266-5273.

PÉREZ, J., M. MENÉNDEZ, S. LARRAÑAGA \& J. POZO. 2011. Inter- and Intra-Regional Variability of Leaf Litter Breakdown in Reference Headwater Streams of Northern Spain: Atlantic versus Mediterranean Streams. International Review of Hydrobiology, 96(1): 105-117.

PÉREZ, J., E. DESCALS \& J. POZO. 2012. Aquatic Hyphomycete Communities Associated with Decomposing Alder Leaf Litter in Reference Headwater Streams of the Basque Country (Northern Spain). Microbial Ecology, 64: 279-290.

PÉREZ, J., A. BASAGUREN, E. DESCALS, A. LARRAÑAGA \& J. POZO. 2013. Leaf-Litter Processing in Headwater Streams of Northern Iberian Peninsula: Moderate Levels of Eutrophication Do Not Explain Breakdown Rates. Hydrobiologia, 718(1): 41-57.

POZO, J. 1993. Leaf-Litter Processing of Alder and Eucalyptus in the Aguera Stream System (North
Spain).1. Chemical Changes. Archiv für Hydrobiology, 127: 299-317.

POZO, J., E. GONZÁLEZ, J. DÍEZ \& A. ELÓSEGUI. 1997. Leaf-Litter Budgets In Two Contrasting Forested Streams. Limnetica, 13(2): 77-84.

POZO, J., A. BASAGUREN, A. ELOSEGI, J. MOLI-

NERO, E. FABRE \& E. CHAUVET. 1998. Afforestation with Eucalyptus globulus and Leaf Litter Decomposition in Streams of Northern Spain. Hydrobiologia, 373: 101-109.

POZO, J., J. CASAS, M. MENÉNDEZ, S. MOLLÁ, I. AROSTEGUI, A. BASAGUREN, C. CASADO, E. DESCALS, J. GARCÍA-AVILÉS, J. M. GONZÁLEZ, A. LARRAÑAGA, E. LÓPEZ, M. LUSI, O. MOYA, J. PÉREZ, T. RIERA, N. ROBLAS \& M. J. SALINAS. 2011. Leaf-Litter Decomposition in Headwater Streams: A Comparison of the Process Among Four Climatic Regions. Journal of North American Benthological Society, 30(4): 935-950.

REECE, P. F. \& J. S. RICHARDSON. 1999. Biomonitoring With the Reference Condition Approach for the Detection of Aquatic Ecosystems at Risk. En: Proceeding of a Conference on the Biology and Management of species and Habitats at Risk (Ed. L. M. Darling),. Ministry of Environment, Lands and Parks, Victoria, B. C. \& University College of the Cariboo, Kamloops, B. C.

REIS, J., R. A. BAILEY, D.M. PERKINS, A. PLUCHINOTTA \& G. WOODWARD. 2011. Testing Effects of Consumer Richness, Evenness and Body Size on Ecosystem Functioning. Journal of Animal Ecology, 80: 1145-1154.

RIIPINEN, M. P., J. DAVY-BOWKER \& M. DOBSON. 2009. Comparison of Structural and Functional Stream Assessment Methods to Detect Changes in Riparian Vegetation and Water pH. Freshwater Biology, 54: 2127-2138.

RIIPINEN, M. P., T. FLEITUCH, S. HLADYZ, G. WOODWARD, P. GILLER \& M. DOBSON. 2010. Invertebrate Community Structure and Ecosystem Functioning in European Conifer Plantation Streams. Freshwater Biology, 55: 346-359.

SAMPAIO, A., P. RODRÍGUEZ-GONZÁLEZ, S. VARANDAS, R. M. CORTES \& M.T. FERREIRA. 2008. Leaf Litter Decomposition in Wwestern Iberian Forested Wetlands: Lentic versus Lotic Response. Limnetica, 27(1): 93-106.

SANDIN, L. \& A. G. SOLOMINI. 2009. Freshwater Ecosystem Structure-Function Relationships: 
From Theory to Application. Freshwater Biology, 54: 2017-2024.

TACHET, H., P. RICHOUX, M. BOURNAUD \& F. USSEGIO-POLATERRA. 2002. Invertébrés d'eau douce. Systematique, biologie, écologie. CNRS Editions, París.

TIEGS, S. D., F. D. PETER, C. T. ROBINSON, U. UEHLINGER \& M. O. GESSNER. 2008. Leaf Decomposition and Invertebrates Colonization Responses to Manipulated Litter Quantity in Streams. Journal of the North American Benthological Society, 27: 321-331.

TIEGS, S. D., O. AKINWOLE \& M. O. GESSNER. 2009. Litter Decomposition Across Multiple Spatial Scales in Stream Networks. Oecologia, 161: 343-351.

WALLACE, J. B., S. L. EGGERT, J. L. MEYER \& J. R. WEBSTER. 1997.Multiple Trophic Levels of a Forest Stream Linked to Terrestrial Litter Inputs. Science, 277: 102-147.

WARD, G. M. \& D. R. WOODS. 1986. Lignin and Fiber Content of FPOM Generated by the Shredders
Tipula abdominalis (Diptera: Tipulidae) and $T a$ llaperla cornelia (Needham \& Smith) (Plecoptera: Peltoperlidae). Archiv für Hydrobiologie, 107: 545-562.

WEBSTER, J. R. \& E. F. BENFIELD. 1986. Vascular Plant Breakdown in Freshwater Ecosystems. Annual Review of Ecology and Systematics, 17: 567594.

WEBSTER, J. R., E. F. BENFIELD, T. P. EHRMAN, M. A. SCHAEFFER, J. L. TANK, J. J. HUTCHES \& D.J. D'ANGELO. 1999. What Happens to Allochthonous Material that Falls into Streams? A Synthesis of New Published Information from Coweeta. Freshwater Biology, 41: 687-705.

YOUNG, R. G., C. D. MATTHAEL \& C. R. TOWNSEND. 2008. Organic Matter Breakdown and Ecosystem Metabolism: Functional Indicators for Assessing River Ecosystem Health. Journal of the North American Benthological Society, 27: 605-625.

ZAR, J. H. 2010. Bioestatistical Analysis. (5 ${ }^{\text {th }}$ Edt.). Pearson Prentice Hall. Londres. 